\title{
30. ANTARCTIC DEEP-SEA BASALT, SOUTHEAST INDIAN OCEAN AND BALLENY BASIN, DSDP LEG 28
}

\author{
A.B. Ford, U.S. Geological Survey, Menlo Park, California
}

\begin{abstract}
Tholeiitic basalt of apparent extrusive origin was recovered at Sites 265 and 266 on the south flank of the Southeast Indian Ridge, at Site 267 in the South Indian Basin, and at Site 274 near Balleny Basin. Inferred age of basalts decreases away from the ridge axis. 01normative tholeiite, containing microphenocrystic olivine, occurs at Site 265 , about $500 \mathrm{~km}$ from the ridge axis. At Site $266,300 \mathrm{~km}$ farther down the ridge flank, fresh aphyric glass is Pl-01 normative and has $0.47 \% \mathrm{~K}_{2} \mathrm{O}$. Devitrified glasses at Site 266 are slightly undersaturated to slightly oversaturated tholeiites. Site 267 basalts are slightly oversaturated tholeiites containing plagioclase microphenocrysts. Although many Leg 28 rocks are Q normative, after reduction of excess $\mathrm{Fe}_{2} \mathrm{O}_{3}$ only those at Site 274 retain appreciable $\mathrm{Q}$. The cored rocks at the four sites are characterized by relatively high $\mathrm{K}_{2} \mathrm{O}$, but most other major and trace elements approximate mid-ocean ridge basalt (MORB) values. Basalts at Sites 265,266 , and 267 are probable ridge-flank and ocean-basin flows. Those from Site 274 are holocrystalline plagioclase-phenocrystic probable flow breccias that may relate to an early stage of Balleny Islands volcanism, laer stages of which produced alkali-olivine basalts that are exposed on the islands.

Most Leg 28 basalts are altered, some to a considerable degree, as indicated by generally high $\mathrm{Fe}$ oxidation, high total $\mathrm{H}_{2} \mathrm{O}$, presence of secondary minerals, and commonly low bulk densities. Average $\mathrm{H}_{2} \mathrm{O}$ contents at most sites are less than average for all DSDP basalt. Alteration resulted in apparent increase only in $\mathrm{K}$ among major elements, and in little significant change in minor elements, including $\mathrm{U}$, in contrast to results reported for Mid-Atlantic Ridge basalt.
\end{abstract}

\section{INTRODUCTION}

\section{Location}

Basalt was recovered at 4 of the 11 sites on Leg 28 of the Deep Sea Drilling Project (Figure 1). Three of the four sites lie along a line approximately normal to the axis of the Southeast Indian Ridge that lies between Australia and Antarctica. Sites 265 and 266 are on the south flank of the ridge about $500 \mathrm{~km}$ and $800 \mathrm{~km}$, respectively, from the ridge crest, and Site 267 lies farther south in the deep South Indian Basin about 600 $\mathrm{km}$ north of the continental shelf of Wilkes Land, Antarctica. The fourth, Site 274, lies near Balleny Basin just east of the Southeast Indian Ocean and near the lower continental rise of northern Victoria Land, Antarctica, about $250 \mathrm{~km}$ north-northeast of Cape Adare.

\section{Occurrence}

Relationships for distinguishing whether the basalt is (1) layer 2 sea-floor basement, (2) an intrasediment flow, or (3) an intrusive into soft or lithified sediment were not found in the core at any site. The rocks show many similarities with mid-ocean ridge basalt (MORB) elsewhere, but they also show significant differences. The progressive age increase of basal sediments away from the ridge, from middle Miocene at Site 265 to middle Oligocene at Site 267, is compatible with alternative 1 above. However, as the basalt was penetrated only superficially, the other alternatives cannot be discounted.

Recovery at all sites in the southeast Indian Ocean was low, on the order of $20 \%$ or less, and at no site was the original contact of sediment against basalt recovered intact. Sediment and basalt form separate core pieces; as much as 7 or 8 meters of sediment originally intervening between lowest recovered sediment fragments and highest basalt may have been lost by drilling at high pressures upon encountering hard basalt. Furthermore, much basalt was lost by drilling, so the original relationships between many adjoining core fragments of basalt are uncertain, making questionable any attempt to distinguish individual flow or cooling units. The uppermost cored basalt at Sites 265 and 267 is glassy and variolitic, becoming more crystalline downward but with occasional intermixing of crystalline and glassy fragments. This relationship suggests that pillowsurfaced flows were penetrated.

At each site, induration of the sediments grades downward from soft to stiff to semilithified or lithified just above basalt, which might be interpreted as 


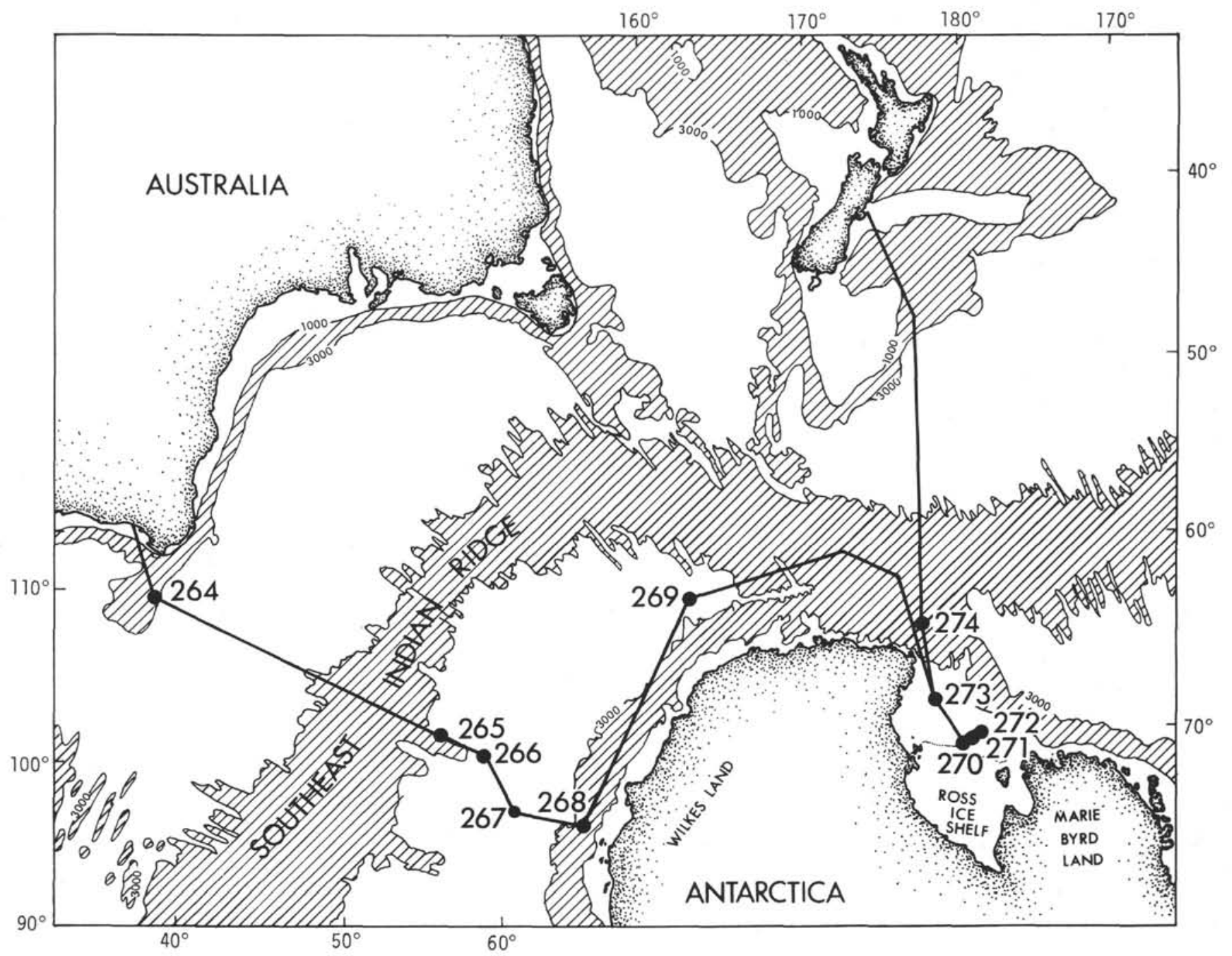

Figure 1. Track of Leg 28 of Glomar Challenger, showing sites with basalt recovery.

evidence for intrusive emplacement of the basalt. However, the lowest recovered sediment though relatively indurated shows no textural evidence of metamorphic recrystallization, and microfossils if present retain original details of fine structure. Studies elsewhere show that contact metamorphic effects of submarine basaltic intrusions may be surprisingly small or absent (Garrison et al., 1973). On the other hand, lithification may result from precipitation of cements derived from low-temperature alteration or weathering of basaltic glass (Garrison et al., 1973). Accordingly, neither lithification nor apparent absence of metamorphic effects conclusively indicates either an intrusive or extrusive origin, respectively, of the basalt. Pillowlike structures might form by either origin. In fact, the distinction between a submarine flow and an intrusion becomes arbitrary in cases where a flow plows beneath and "intrudes" cold basal sediments as at Site 205 of Leg 21 where probable pillows are intermixed with limestone (Churkin and Packham, 1973). Such intermixtures, however, were not seen in Leg 28 cores.

\section{Analytical Methods}

Major-element chemical analyses were made by Paul L.D. Elmore using rapid-rock analytical methods described in U.S. Geological Survey Bulletin 1144-A and supplemented by atomic-absorption analysis. Minor-element abundances were determined by a variety of methods. Th and $U$ were determined by H.T. Millard, P.J. Aruscavage, Ardith Bartel, and R.A. Zielinski using a delayed neutron system. S was determined by L. Shapiro using the method of solubility in aqua regia. $\mathrm{Au}$ and $\mathrm{Ag}$ were analyzed by Louis $\mathrm{J}$. Schwarz by neutron-activation methods using fire assay for radiochemical separations as described in U.S. Geological Survey Circular 599. Ce, Cr*, Eu, Hf, Nd, $\mathrm{Sb}, \mathrm{Ta}, \mathrm{Th}^{*}$, and $\mathrm{Yb}^{*}$ were determined by Louis $\mathrm{J}$. Schwarz by neutron-activation methods. The reported radioactivation and radiochemical results are averages using different photopeaks and(or) several counting runs; those reported to in two or more significant figures including at least the tenths digit have an overall ac- 
curacy of $\pm 10 \%$, and the others are assumed to have an accuracy to $\pm 50 \%$ owing to low counting rate, heavy interference, and(or) concentration below detection limit. All other minor elements were determined by R.E. Mays using quantitative spectrographic analysis. Spectrographic results are reported to two significant figures and have an overall accuracy of $\pm 15 \%$ except that they are less accurate near the limits of detection where only one digit is intended. $\mathrm{Cr}$, Th, and $\mathrm{Yb}$, in different sample splits, were analyzed by both neutron activation (indicated by ${ }^{*}$ ) and spectrographic or other methods. Powder densities were measured by pycnometer by $\mathrm{L}$. Shapiro.

Alteration has a marked effect on normative compositions of some of the basalts. Most pronounced is a high degree of $\mathrm{Fe}$ oxidation which plays a major role in partitioning of components into normative minerals, particularly Q, Ne, and femic minerals (Coombs, 1963). CIPW norms have been computed both for original analyses and for analyses normalized $\mathrm{H}_{2} \mathrm{O}$-free and with all $\mathrm{Fe}_{2} \mathrm{O}_{3}$ in excess of $1.5 \%$ recalculated as $\mathrm{FeO}$. It is assumed that $111.5 \%$ is the primary amount of $\mathrm{Fe}_{2} \mathrm{O}_{3}$ in these basalts (Coombs, 1963; Shido et al., 1971). Adjusted norm values are shown in parentheses in the Tables.

Alteration has a marked effect on normative compositions of some of the basalts. Most pronounced is a high degree of $\mathrm{Fe}$ oxidation which plays a major role in partitioning of components into normative minerals, particularly Q, Ne, and femic minerals (Coombs, 1963). CIPW norms have been computed both for original analyses and for analyses normalized $\mathrm{H}_{2} \mathrm{O}$-free and with all $\mathrm{Fe}_{2} \mathrm{O}_{3}$ in excess of $1.5 \%$ recalculated as $\mathrm{FeO}$. It is assumed that $1.5 \%$ is the primary amount of $\mathrm{Fe}_{2} \mathrm{O}_{3}$ in these basalts (Coombs, 1963; Shido et al., 1971). Adjusted norm values are shown in parentheses in the Tables.

Terms and symbols used in this report are defined as follows:

$$
\begin{aligned}
& \mathrm{FeO}^{*}=\mathrm{FeO}+0.9 \mathrm{Fe}_{2} \mathrm{O}_{3} \\
& \text { oxidation index }=100 \mathrm{Fe}_{2} \mathrm{O}_{3} /\left(\mathrm{Fe}_{2} \mathrm{O}_{3}+\mathrm{FeO}\right) \\
& \text { mafic index }=100\left(\mathrm{FeO}+\mathrm{Fe}_{2} \mathrm{O}_{3}\right) /\left(\mathrm{FeO}+\mathrm{Fe}_{2} \mathrm{O}_{3}\right. \\
& \quad+\mathrm{MgO}) \\
& \begin{array}{c}
\text { D.I. }=\text { differentiation index of Thornton and } \\
\text { Tuttle (1960) }
\end{array}
\end{aligned}
$$

Sample numbers in Tables 1-16 are given in order of increasing depth measured from the top of the basalt recovered in each core length. In these incomplete cores, depths given may not be original ones (see discussion in Laughton, Berggren, et al., 1972). Analytical data are designated in various figures using letter symbols given in the tables.

Modes were determined by counting approximately 1000 points covering the areas of standard-size thin sections. Plagioclase compositions were determined optically by the universal-stage method of Uruno (1963) combined with the extinction-curve method of Tobi (1963). Colors and color symbols used are those of the Rock Color Chart of the Geological Society of America (1963).

\section{Alteration}

The highly significant chemical changes in marine basalt, involving both major and minor elements, that accompany alteration by prolonged exposure to seawater clearly require caution in interpreting original magma type from rock chemistry (Bass et al., 1973; Thompson, 1973). Few Leg 28 basalt analyses show less than $2 \%$ total $\mathrm{H}_{2} \mathrm{O}$ and oxidation index lower than 40 . Thus major chemical changes by alteration can be expected based on the data of Cann (1971). Chemical and mineralogic alteration generally results in a decrease in density of sea-floor basalt to depths locally as great as several hundred meters, indicating that alteration processes can operate to considerable depths even beneath accumulations of marine sediment (Salisbury and Christensen, 1973). In an environment of high $\mathrm{CO}_{2}$ activity, as under a pile of calcareous sediments, the principal chemical changes are a gain of $\mathrm{H}_{2} \mathrm{O}, \mathrm{K}$, and $\mathrm{Na}$; loss of $\mathrm{Ca}$, mainly from clinopyroxene; and oxidation of $\mathrm{Fe}$ (Bass et al., 1973). Although variable, the common net effect of alteration is an increase in apparent "alkalinity" of major-element chemistry, according to Bass et al., (1973).

Fresh, nonhydrated, crystal-free glass that may closely approximate original magma composition is rare in Leg 28 cores. The only such fresh glass analyzed is Sample 266-23-1-4 (Tables 5-8). The Hy-01 normative composition of the rock indicates it is an olivine tholeiite (Yoder and Tilley, 1962). Comparative freshness of this glass is indicated by its lower than average $\mathrm{H}_{2} \mathrm{O}$ content and considerably lower oxidation index than the other 20 analyzed samples. The glass, however, has higher $\mathrm{K}_{2} \mathrm{O}$ content than fresh abyssal mid-ocean-ridge tholeiite elsewhere (Miyashiro et al., 1969; Cann, 1971). In the absence of any visible or other analytical evidence of alteration, the high $\mathrm{K}_{2} \mathrm{O}$ content is assumed to be a primary characteristic of the rock. Basalt at Site 215, Leg 22, in the central Indian Ocean, also is distinctly more "alkaline" than basalts typical of active mid-ocean ridges (Thompson et al., 1974). Comparison of analyses of the Site 266 fresh glass and those of palagonitized, devitrified, and variolitic glasses from nearby in the same hole suggests that $\mathrm{K}, \mathrm{Na}$, and $\mathrm{Ca}$ transfer has been minimal though analyses overall indicate that $\mathrm{K}$ increases with oxidation (Figure 2). The major general changes during alteration at Site 266 were oxidation of $\mathrm{Fe}$ and gain of $\mathrm{H}_{2} \mathrm{O}$ and, in some rocks, gain of $\mathrm{CO}_{2}$. Alteration effects were probably similar at other Leg 28 sites but are more uncertain in the absence of fresh material for comparison.

Studies of Miyashiro et al. (1969) show that unweathered abyssal tholeiite has $\mathrm{Fe}_{2} \mathrm{O}_{3}$ in the range $0.8 \%$ $2.0 \%, \mathrm{H}_{2} \mathrm{O}+$ in the range $0.5 \%-0.9 \%$, and $\mathrm{H}_{2} \mathrm{O}-$ in the range $0.0 \%-0.3 \%$. Except for glass (266-23-1-4) all Leg 28 analyzed basalts have compositions outside these limits, and thus are apparently altered to some degree, some being very highly altered (Figure 3 ). However, as indicated by total $\mathrm{H}_{2} \mathrm{O}$ content, the degree of alteration of basalt at Sites $265-267$ is less than the overall average for 


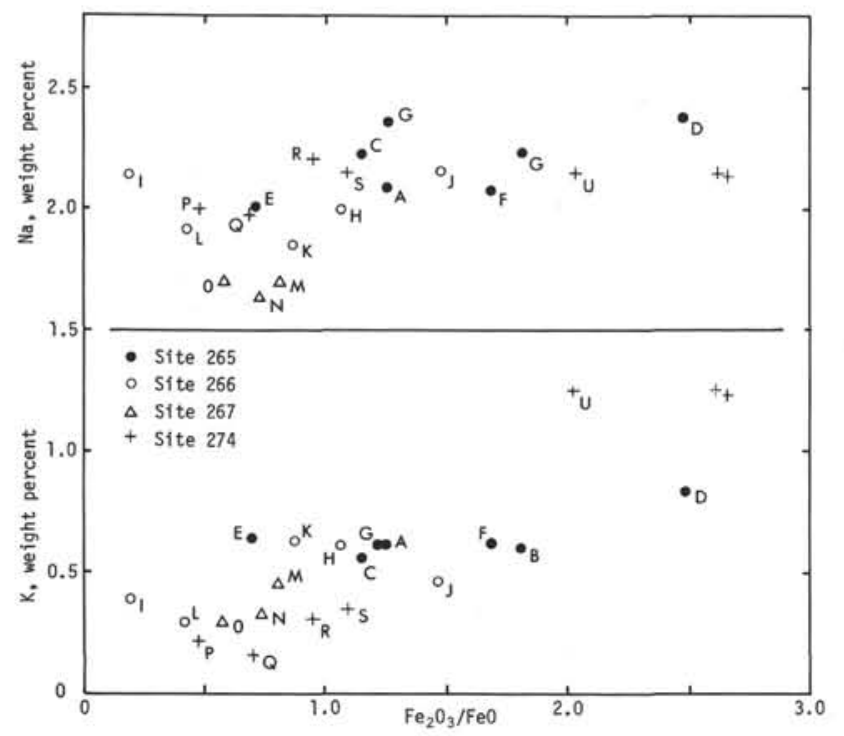

Figure 2. Relation of $\mathrm{K}$ and $\mathrm{Na}$ to oxidation of Leg 28 basalts.
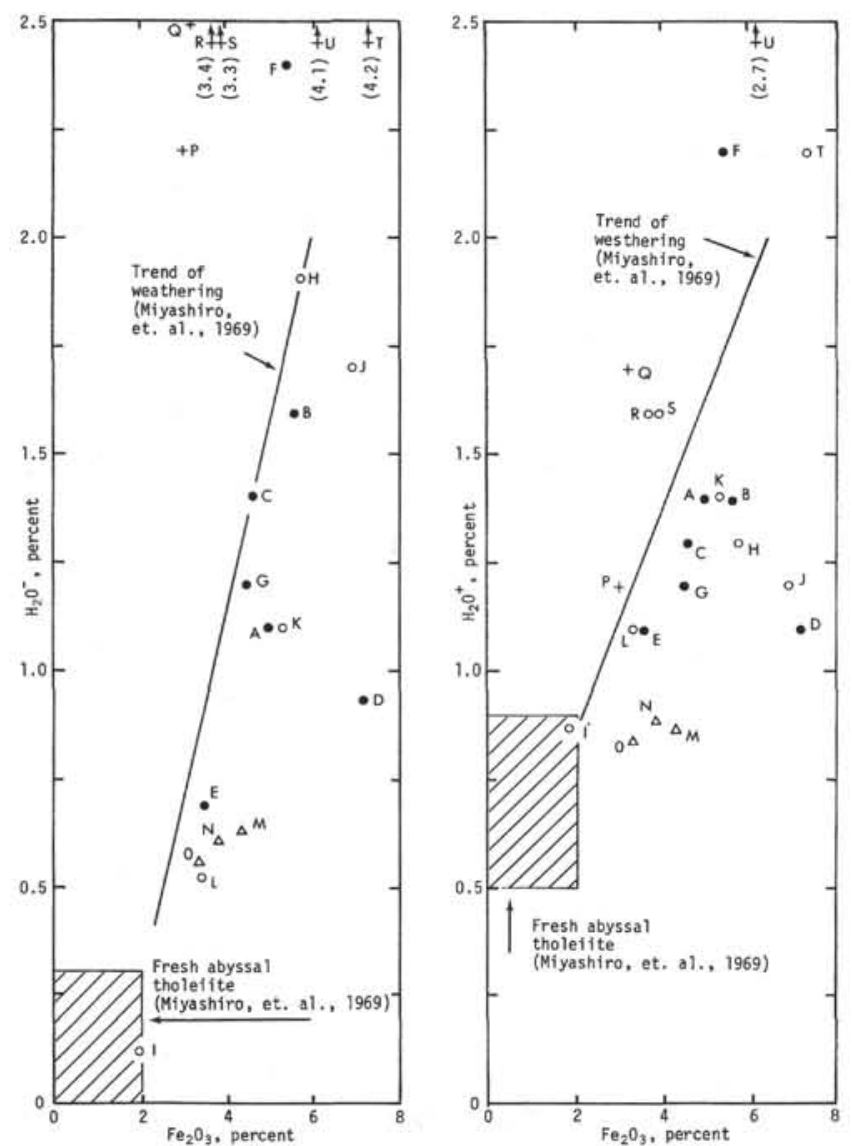

Figure 3. Relation of $\mathrm{H}_{2} \mathrm{O}$ - and $\mathrm{H}_{2} \mathrm{O}+$ to $\mathrm{Fe}_{2} \mathrm{O}_{3}$ in Leg 28 basalt, compared to weathering trend of Mid-Atlantic Ridge tholeiite shown by line (Miyashiro et al., 1969). Lined areas indicate unweathered Mid-Atlantic Ridge tholeiites. Letters designate analyses in Tables 1-16. all DSDP basalt sampled up to Leg 34 (Hart, S.R., Yeats, R.S., et al., 1974), and average Site 267 basalt, with only $1.47 \%$ total $\mathrm{H}_{2} \mathrm{O}$, is much less than the overall average. Average Site 274 basalt with $5.2 \%$ total $\mathrm{H}_{2} \mathrm{O}$ is higher than the overall DSDP average.

Relative degree of alteration of Leg 28 basalts can be inferred from Figure 3 and from $\mathrm{Fe}$ oxidation (Figures 4,5 ) and from density differences (Figure 6). Progressive alteration results in increasing content of secondary products and therefore generally decreasing density which for some other DSDP sites is postulated to be related to progressive age increase away from a spreading ridge axis (Salisbury and Christensen, 1973). Densities of Leg 28 basalts are listed in Tables 4, 8, 12, 16. Leg 28 basalt densities generally decrease with alteration (Figure 6), those of Site 274 apparently decreasing along a different trend from those of the southeast Indian Ocean sites. Other factors such as glass content or compositional differences obviously contribute to density differences and produce the scatter in Figure 6; the fresh glass sample (266-23-1-4), for example, has the lowest oxidation index, 17, and lower than average density, $2.77 \mathrm{~g} / \mathrm{cc}$. Except for Site 267, average densities decrease inversely with inferred ages (Figure 6).

$\mathrm{Fe}$ oxidation significantly affects normative mineralogy and norm-based rock classification. In terms of relative molecular proportions of $\mathrm{Q}, \mathrm{Di}, \mathrm{Hy}$, and $\mathrm{Ol}$, the reduction of excess $\mathrm{Fe}_{2} \mathrm{O}_{3}$ to an assumed original value of $1.5 \%$ can result in appreciable decrease in $\mathrm{Q}$, if originally present, and corresponding changes in $\mathrm{Hy}$ and Ol (Coombs, 1963). Di remains unchanged except in rare Leg 28 recalculated norms containing $\mathrm{Ne}$. Tables 2 , $6,10,14$, and 18 and Figure 7 show the extent of such changes. True primary compositions probably are between the given values in the tables and lie between endpoints of lines in Figure 7. Norm recalculations generally yield significant changes only in Q, En, Fs, Ol, and $\mathrm{Mt}$, the amounts depending on the degree of $\mathrm{Fe}$ oxidation. The oxidation appears to have had the net effect of decreasing apparent "alkalinity" as shown in Ne-DiOl-Q plots of Figure 7 after Coombs (1963).

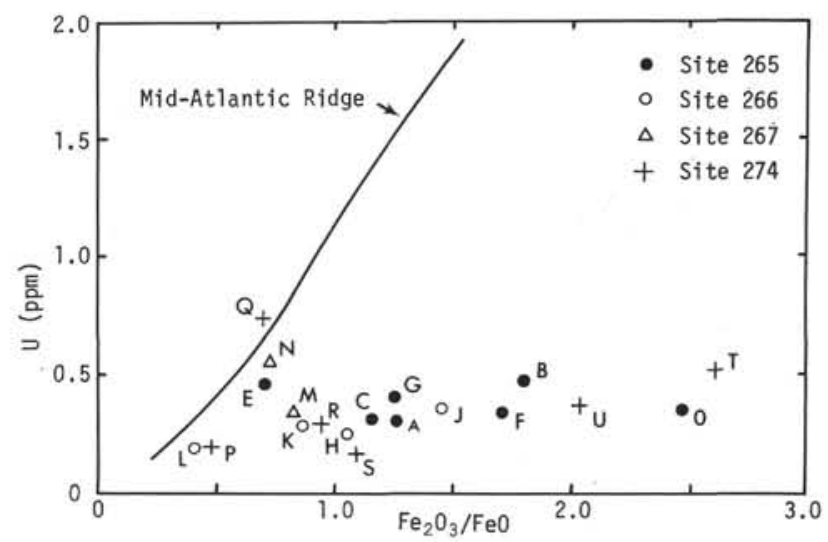

Figure 4. Relation of $U$ to oxidation of Leg 28 basalt. Line shows approximate distribution of values of Aumento (1971). 


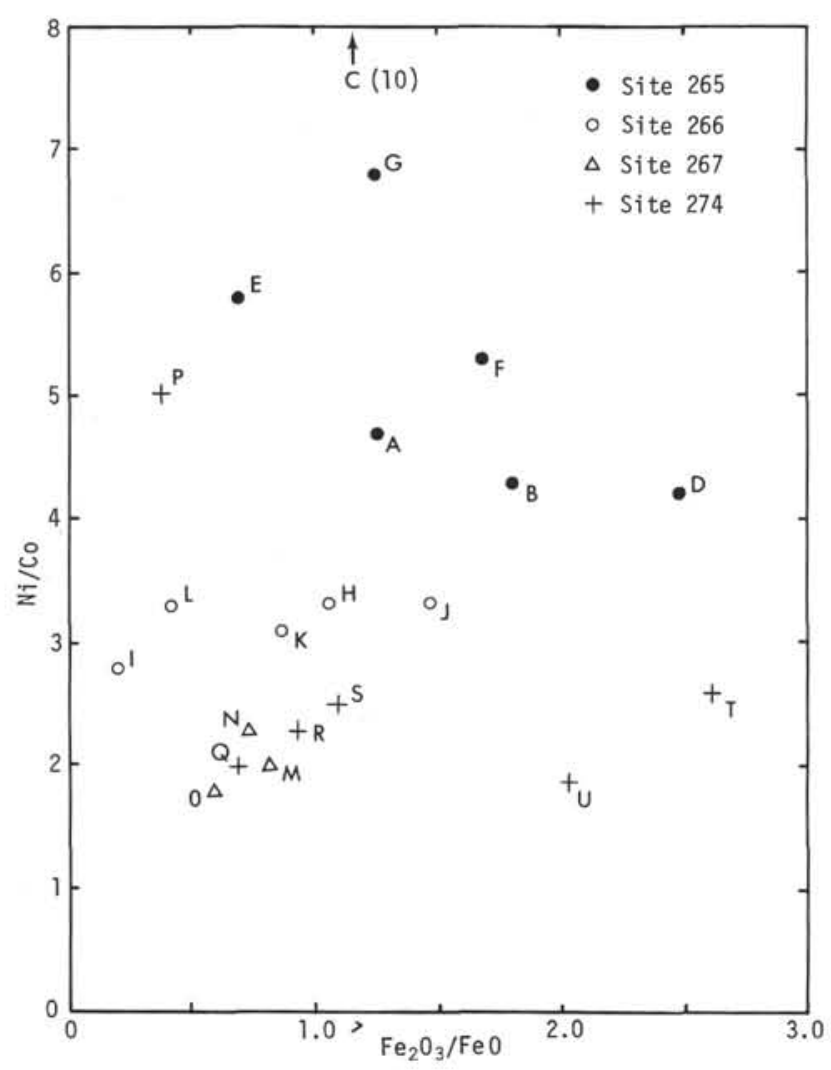

Figure 5. Relation of Ni/Co to oxidation of Leg 28 basalt.

If density (Salisbury and Christensen, 1973) and oxidation index and $\mathrm{H}_{2} \mathrm{O}$ contents (Cann, 1971; Miyashiro et al., 1969) are reliable indicators of alteration, they should vary downward in a rather systematic manner as a result of progressive penetration of the zone of alteration. Densities should generally increase downward and oxidation and $\mathrm{H}_{2} \mathrm{O}$ decrease. These indicators vary as expected only at Site 267. At Sites 265 and 266 they show little systematic variation, and at Site 274 they seem to show a reverse tendency. Obviously, factors such as differences in lithology, including variations in crystallinity, and presence of fractures allowing local penetration of solutions have strongly influenced differential alteration of these rocks. Veinlets of calcite, smectite, and zeolite in some rocks (Figure 8) have highly altered border zones and clearly have been channelways for solutions. Differences in crystallinity are important because glassy and crystalline basalts respond differently to low-temperature alterations by saline pore solutions (Melson, 1973).

\section{SITE 265, SOUTHEAST INDIAN RIDGE}

\section{General}

Site 265 on the south flank of the Southeast Indian Ridge at $53^{\circ} 32.45^{\prime} \mathrm{S}, 109^{\circ} 56.74^{\prime} \mathrm{E}$ is in a water depth of 3582 meters. Basalt was encountered at a subbottom depth of 444.5 meters and was cored continuously to 462 meters. Recovery of basalt was only $20 \%$, mostly as small fragments $3-10 \mathrm{~cm}$ long and totaling about 1.5 meters in Core 17 and 2.1 meters in Core 18 in addition to material in core catchers.

Oceanic basement at the site is inferred from magnetic lineations to be 12-15 m.y. old. The sampled basalt underlies clay- and diatom-bearing nanno ooze and chalk of early middle-Miocene age (see Site 265 paleontology report, this volume). Only a single small piece of poorly consolidated nanno chalk was recovered in Core 17 above fragments of basalt.

\section{Petrography}

Most of the upper 1.5 meters of recovered basalt, in Core 17 , is medium gray devitrified glass containing a few percent olivine microphenocrysts in a variolitic matrix of finely radiating, plumose plagioclase interleaved with brownish and translucent unidentified microcrystalline material. The well-developed form, though small, of olivine indicates that it is the only liquidus phase in the glassy rocks. Vesicles and carbonate-filled amygdules are small, generally between 1 and $3 \mathrm{~mm}$ in size, and make up only $1 \%$ or less of the upper $40 \mathrm{~cm}$ of core. Below that they gradually increase downward in size and amount, commonly reaching $3 \%$ $5 \%$ and locally as much as $10 \%$.

The transition from chiefly devitrified glass to holocrystalline basalt, which extends to the base of the
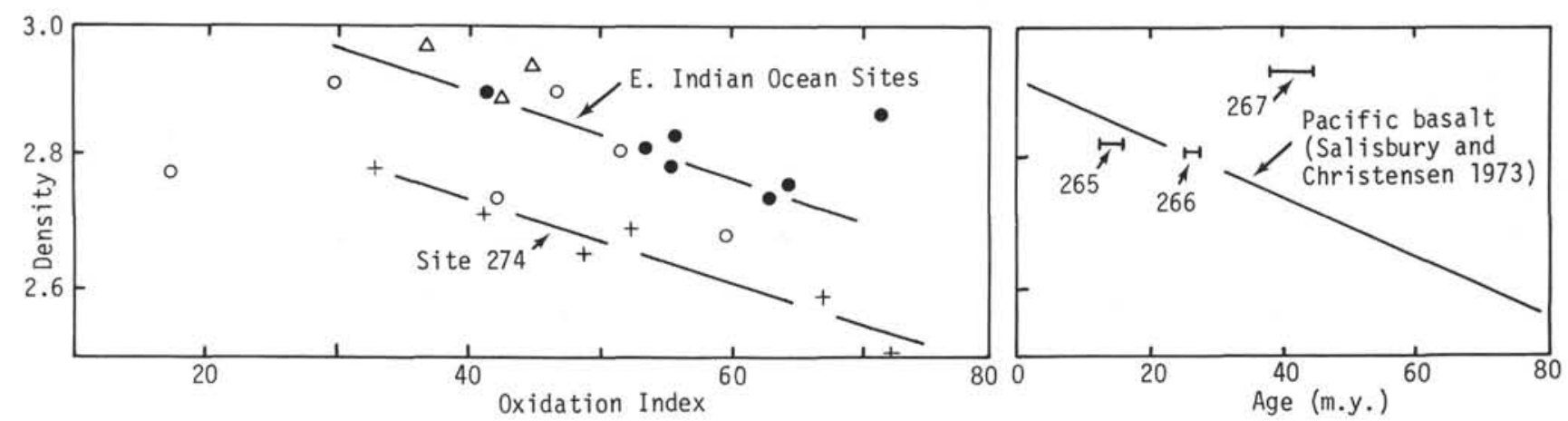

Figure 6. Relations of density to oxidation index of Leg 28 basalt and to crustal ages inferred from magnetic lineations. Length of bars in (b) shows uncertainty of ages and is queried for Site 274 in absence of magnetic lineations. Density values in (b) are site averages. Letter I, in (a) shows fresh glass, 266-23-1-4. 


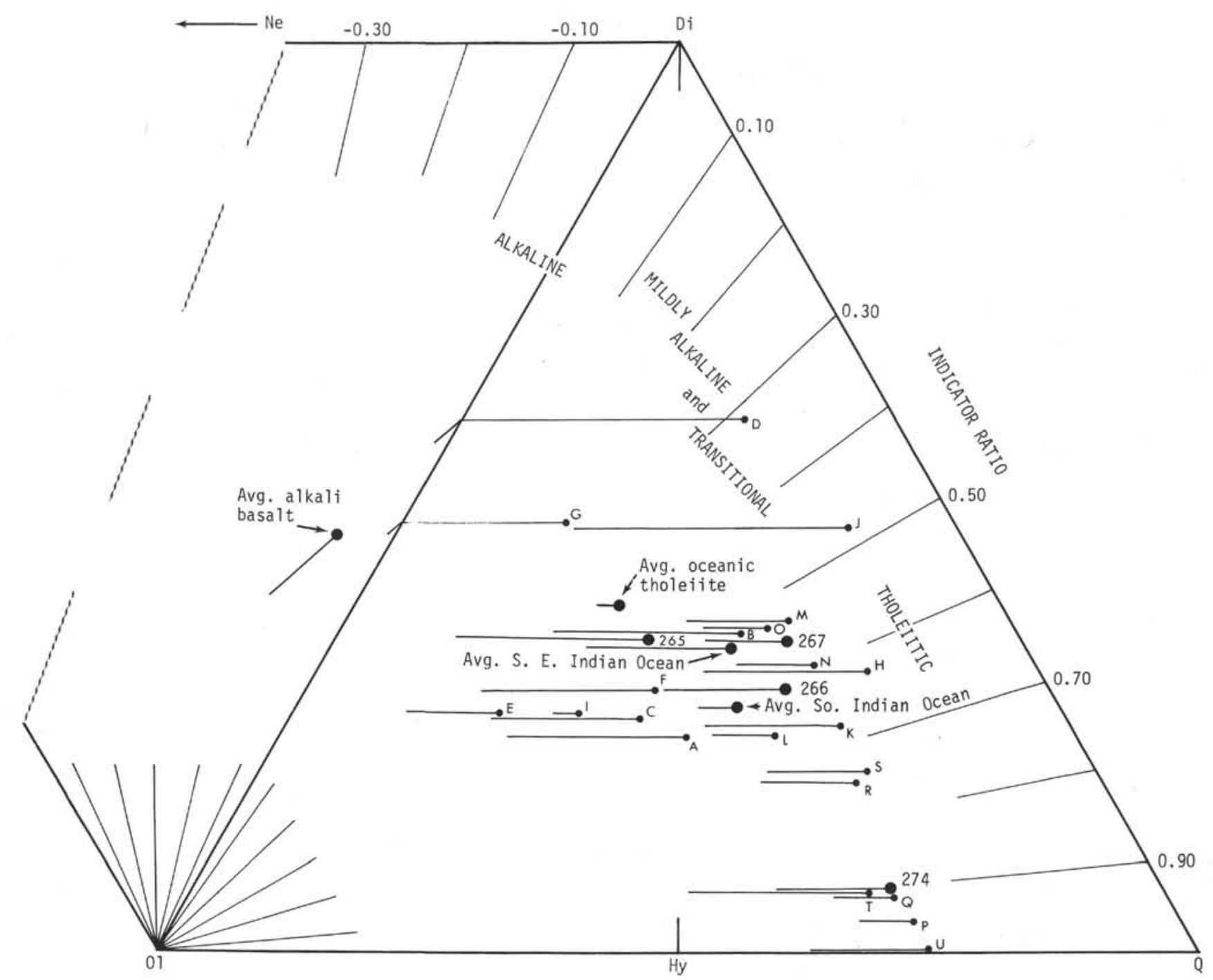

Figure 7. Ne-Di-O1-Q diagram, after Coombs (1963), showing plots of Leg 28 rocks, in molecular proportions, compared to average southern Indian Ocean basalt of Kempe (1973) and to average oceanic tholeiite and alkali basalt of Engel et al. (1965). Leg 28 averages identified by site numbers at points.

hole, occurs between about 120 and $140 \mathrm{~cm}$ depth in Core 17. Based on the presence of an intermixture of fragments of devitrified, variolitic glass and a few fragments of nearly holocrystalline basalt, which possibly represent pillow centers, the upper $120 \mathrm{~cm}$ is believed to represent the pillowed surface of an underlying nearly holocrystalline basalt flow. Several small fragments of olivine-bearing, undevitrified black glass at $60 \mathrm{~cm}$ depth in Core 17 probably are remains of a pillow rim. They are the only apprently fresh glasses recovered from the hole; but the glass is surrounded by colloformstructured palagonitic rims a few millimeters wide bordered by a zeolitized tuffaceous(?) matrix. No recovered core lengths, however, show complete transition from glassy rim to holocrystalline core of a pillow.

Below about $120 \mathrm{~cm}$ depth in Core 17, and continuing to the base of the hole, the basalt is nearly holocrystalline and only locally microporphyritic. Modes of chemically analyzed samples are given in Table 4. Textures are mostly intergranular to intersertal, but vary in places to microdiabasic. The basalt consists chiefly of lath-shaped plagioclase, and slightly lesser amounts of clinopyroxene. Most plagioclase (Figure 9) shows weak and gradational normal zoning from cores that are sodic bytownite or calcic labradorite (An65-73) to rims of intermediate to sodic labradorite. Preliminary results of electron microprobe analyses by L.C. Calk, U.S. Geological Survey, indicate that the clinopyroxenes are in the augite-ferroaugite series. Augite crystal centers, of about $\mathrm{Ca}_{47} \mathrm{Fe}_{28} \mathrm{Mg}_{25}$ composition, have $\mathrm{Fe}$ enriched rims of about $\mathrm{Ca}_{40} \mathrm{Fe}_{38} \mathrm{Mg}_{22}$ composition. Olivine is a much lesser constituent, and opaque oxides, carbonate minerals, and other alteration products make up the remainder of most rocks. Olivine, along with plagioclase, is generally nonporphyritic but locally, as in analyzed Sample 265-18-1-6, both occur as euhedral to subhedral microphenocrysts.

No unequivocal evidence was found to indicate that more than a single flow unit was penetrated at this site. Except for a varying degree of crystallinity and altera- 

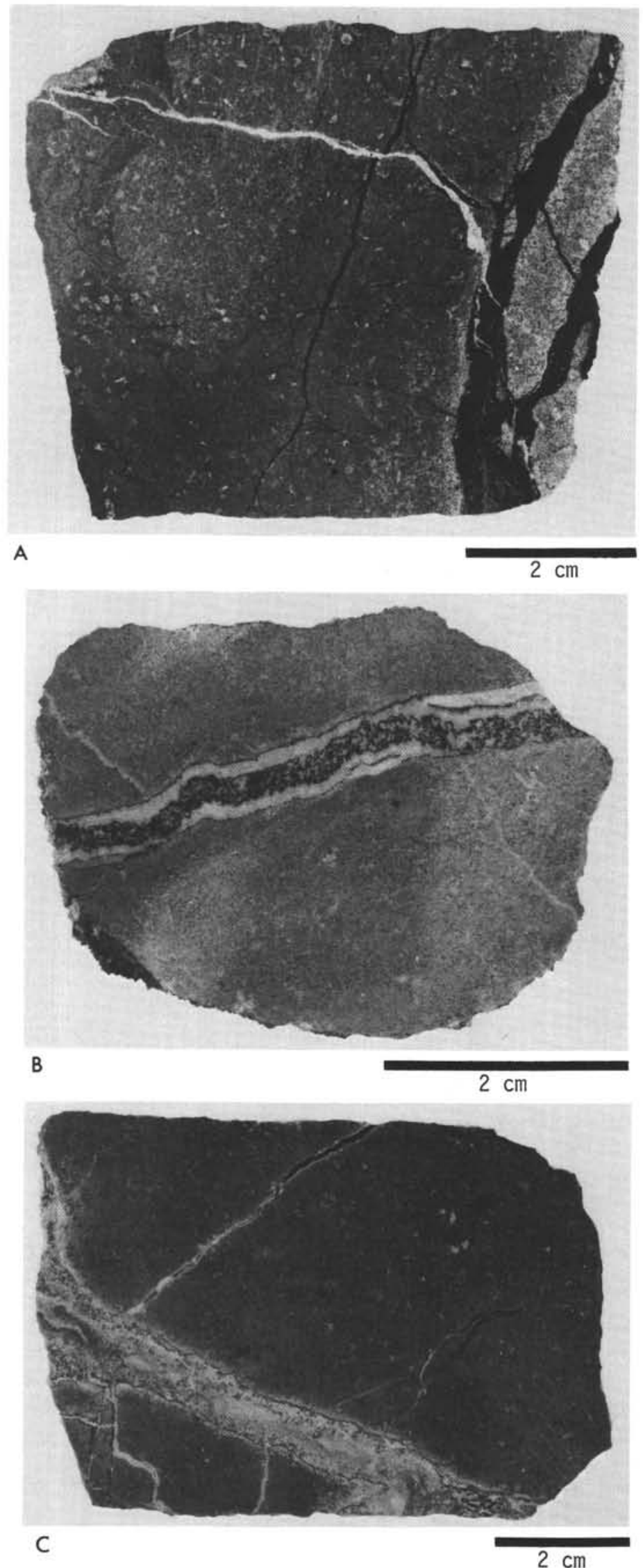

Figure 8. (a) Veinlets of calcite (white) and chlorite (greenish-black) cutting medium dark gray basalt; Core 45, depth $2 \mathrm{~cm}$. (b) Zoned veinlet of zeolite and calcite with chloritic center in medium dark gray plagioclase porphyritic basalt; Core 44, depth $8 \mathrm{~cm}$. (c) Zoned veinlet of calcite, zeolite, and chlorite with calcite-rich center. Blackish to white smaller veinlets are of chlorite or calcite in medium dark gray plagioclase porphyritic basalt; Core 44, depth $15 \mathrm{~cm}$. 


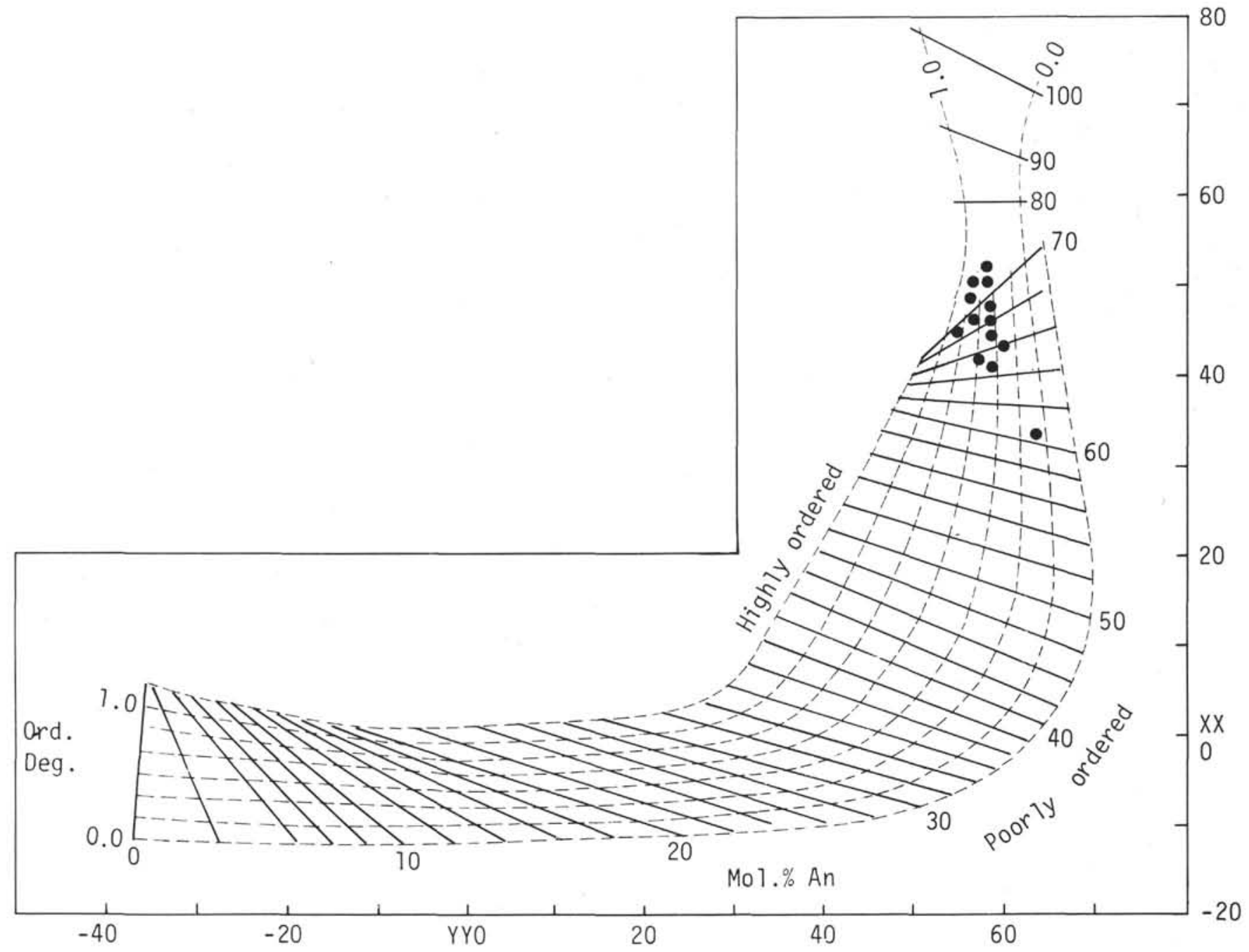

Figure 9. Plots of optical composition and ordering degree of plagioclase, Site 265. Diagram after Uruno (1963). Vertical axis, $X \wedge X$ Kohler angle; horizontal axis, $Y \wedge Y$ Kohler angle. An contents shown on solid diagonal lines.

tion, all rocks are of similar petrographic type. The intermixture of glassy and more crystalline rock fragments in the upper part of Core 17 is interpreted as representing pillow structure rather than indicating multiple flow units.

\section{Chemistry}

Major elements: Major-oxide analyses for seven samples are given in Table 1. The three Core 17 samples are devitrified, variolitic glasses probably from pillows above the main mass of nearly holocrystalline basalt represented by the four Core 18 samples. Other than being on the average slightly more siliceous and containing slightly less $\mathrm{CO}_{2}$, the altered glasses show little discernible difference from the more crystalline basalts. Both groups have about the same mafic index and show about the same degree of hydration and oxidation. Although $\mathrm{Na}_{2} \mathrm{O}$ values for both groups approximate those of MidAtlantic Ridge abyssal tholeiite (Miyashiro et al., 1969) and of average oceanic tholeiite (Engel et al., 1965), $\mathrm{K}_{2} \mathrm{O}$ values are considerably higher. If $\mathrm{K}_{2} \mathrm{O}$ was introduced during alteration of Site 265 basalt, it was taken up to about the same degree by both the glassy rocks and the more crystalline basalt, which seems unlikely. High $\mathrm{K}_{2} \mathrm{O}$ content is probably an original characteristic of these rocks.

Norms of the Site 265 basalts are given in Table 2 . Most norms calculated from unadjusted analyses indicate olivine tholeiite compositions as they contain both $\mathrm{Hy}$ and $\mathrm{Ol}$, but several containing $\mathrm{Q}$ are oversaturated tholeiites (Yoder and Tilley, 1962). Adjustment of analyses for $\mathrm{Fe}$ oxidation results in all norms being free of Q, having lesser $\mathrm{Hy}$ and greater $\mathrm{Ol}$ and, in two rocks, in norms containing minor Ne. Most, if not all, of the rocks were probably originally olivine tholeiites. Normative plagioclase, with compositional range from about $A_{51}$ to $A_{58}$, is less calcic than optically determined compositions (Table 4) probably because of the presence of sodic feldspar in turbid interstitial regions.

Minor elements: Minor elements are listed in Table 3. Other than slightly higher $\mathrm{Au}$ and lower $\mathrm{Cr}$ and $\mathrm{V}$ in the glassy rocks of Core 17 compared to the more crystalline 
TABLE 1

Major-Oxide Analyses of Basalts From Site 265, Southeast Indian Ridge

\begin{tabular}{|c|c|c|c|c|c|c|c|}
\hline $\begin{array}{c}\text { Sample } \\
\text { Depth in Core }(\mathrm{cm}) \\
\text { Plot Symbol }\end{array}$ & $\begin{array}{c}17-1-2 \\
17-25 \\
\text { A }\end{array}$ & $\begin{array}{l}17-1-8 \\
50-57 \\
B\end{array}$ & $\begin{array}{c}17-1-21 \\
109-115 \\
C\end{array}$ & $\begin{array}{c}18-1-3 \\
15-23 \\
\text { D }\end{array}$ & $\begin{array}{c}18-1-6 \\
40-47 \\
E\end{array}$ & $\begin{array}{l}18-2-9 \\
55-60 \\
F\end{array}$ & $\begin{array}{c}18, \mathrm{CC} \\
\mathrm{G}\end{array}$ \\
\hline $\mathrm{SiO}_{2}$ & 48.0 & 48.4 & 48.4 & 47.6 & 47.3 & 47.1 & 47.1 \\
\hline $\mathrm{A}_{2}{ }_{2} \mathrm{O}_{3}$ & 17.7 & 17.0 & 16.5 & 17.3 & 16.2 & 16.0 & 16.5 \\
\hline $\mathrm{Fe}_{2} \mathrm{O}_{3}$ & 5.0 & 5.6 & 4.6 & 7.2 & 3.5 & 5.4 & 4.5 \\
\hline $\begin{array}{l}\mathrm{FeO} \\
\mathrm{MgO} \\
\mathrm{CaO} \\
\mathrm{Na}_{2} \mathrm{O}\end{array}$ & $\begin{array}{l}4.0 \\
7.4 \\
9.8 \\
2.8\end{array}$ & $\begin{array}{r}3.1 \\
6.5 \\
10.4 \\
3.0\end{array}$ & $\begin{array}{l}4.0 \\
8.6 \\
9.4 \\
3.0\end{array}$ & $\begin{array}{r}2.9 \\
4.5 \\
11.5 \\
3.2\end{array}$ & $\begin{array}{r}5.0 \\
10.0 \\
10.2 \\
2.7\end{array}$ & $\begin{array}{l}3.2 \\
8.6 \\
9.6 \\
2.8\end{array}$ & $\begin{array}{r}3.6 \\
7.2 \\
11.6 \\
3.2\end{array}$ \\
\hline $\mathrm{K}_{2} \mathrm{O}$ & 0.75 & 0.74 & 0.68 & 1.0 & 0.77 & 0.64 & 0.75 \\
\hline $\mathrm{H}_{2} \mathrm{O}+$ & 1.4 & 1.4 & 1.3 & 1.1 & 1.1 & 2.2 & 1.2 \\
\hline $\mathrm{H}_{2} \mathrm{O}-$ & 1.1 & 1.6 & 1.4 & 0.93 & 0.69 & 2.4 & 1.2 \\
\hline $\mathrm{TiO}_{2}$ & 1.6 & 1.6 & 1.6 & 1.6 & 1.5 & 1.5 & 1.5 \\
\hline $\mathrm{P}_{2} \mathrm{O}_{5}$ & 0.29 & 0.29 & 0.28 & 0.30 & 0.26 & 0.27 & 0.27 \\
\hline $\begin{array}{l}\mathrm{MnO} \\
\mathrm{CO}_{2}\end{array}$ & $\begin{array}{l}0.16 \\
0.07\end{array}$ & $\begin{array}{l}0.14 \\
0.18\end{array}$ & $\begin{array}{l}0.14 \\
0.07\end{array}$ & $\begin{array}{l}0.18 \\
0.67\end{array}$ & $\begin{array}{l}0.18 \\
0.62\end{array}$ & $\begin{array}{l}0.13 \\
0.05\end{array}$ & $\begin{array}{l}0.16 \\
1.2\end{array}$ \\
\hline Total & 100.1 & 100.0 & 100.0 & 100.0 & 100.0 & 99.9 & 100.0 \\
\hline
\end{tabular}

TABLE 2

CIPW Weight Norms of Rocks From Site $265^{\mathrm{a}}$

\begin{tabular}{|c|c|c|c|c|c|c|c|}
\hline $\begin{array}{l}\text { Sample } \\
\text { Plot } \\
\text { Symbol }\end{array}$ & $17-1-2$ & $17-1-8$ & $17-1-21$ & $\begin{array}{c}18-1-3 \\
\text { D }\end{array}$ & $\begin{array}{c}18-1-6 \\
\text { E }\end{array}$ & $\begin{array}{c}18-2-9 \\
F\end{array}$ & $\begin{array}{c}18, \mathrm{CC} \\
\mathrm{G}\end{array}$ \\
\hline Q & $0.2(-)$ & $1.3(-)$ & - & $1.0(-)$ & - & - & - \\
\hline Or & 4.5 & 4.4 & 4.1 & 6.0 & 4.6 & 3.9 & 4.5 \\
\hline $\mathrm{Ab}$ & 23.9 & 25.8 & 25.8 & 27.3 & 23.0 & 24.3 & 27.4 \\
\hline An & 33.9 & 31.3 & 30.0 & 30.2 & 30.0 & 30.0 & 28.8 \\
\hline $\mathrm{Ne}$ & $-(-)$ & $-(-)$ & $-(-)$ & $-(0.5)$ & $-(-)$ & $-(-)$ & $-(0.4)$ \\
\hline (Wo & $5.4(5.5)$ & $7.6(7.7)$ & $6.3(6.4)$ & $8.8(9.0)$ & $6.4(6.5)$ & $7.0(7.2)$ & $8.4(8.5)$ \\
\hline Di $\{$ En & $4.5(3.4)$ & $6.5(4.7)$ & $5.2(4.2)$ & $7.6(4.4)$ & $4.9(4.4)$ & $6.1(4.7)$ & $7.0(5.4)$ \\
\hline CFs & $0.2(1.8)$ & $-(2.6)$ & $0.3(1.7)$ & $-(4.4)$ & $0.8(1.6)$ & $-(2.0)$ & $0.3(2.5)$ \\
\hline $\int$ En & $14.1(6.2)$ & $9.9(5.4)$ & $13.4(6.2)$ & $3.7(-)$ & $7.3(3.5)$ & $13.9(5.5)$ & $4.6(-)$ \\
\hline${ }^{H y}\left\{\left\{_{F s}\right.\right.$ & $0.7(3.2)$ & $-(3.0)$ & $0.7(2.6)$ & $-(-)$ & $1.2(1.3)$ & $-(2.3)$ & $0.2(-)$ \\
\hline $\mathrm{Ol}\left\{\mathrm{Fo}^{\mathrm{Fo}}\right.$ & $-(6.6)$ & $-(4.7)$ & $2.2(8.2)$ & $-(5.0)$ & $9.0(12.3)$ & $1.4(8.7)$ & 4.6(9.1) \\
\hline lFa & $-(3.8)$ & $-(2.8)$ & $0.1(3.7)$ & $-(5.4)$ & $1.7(4.9)$ & $-(3.9)$ & $0.2(4.6)$ \\
\hline Mt & $7.3(2.2)$ & $5.9(2.3)$ & $6.8(2.2)$ & $5.3(2.2)$ & $5.1(2.2)$ & $6.6(2.3)$ & $6.6(2.2)$ \\
\hline $\mathrm{Hm}$ & $-(-)$ & $1.6(-)$ & $-(-)$ & $3.6(-)$ & $-(-)$ & $1.0(-)$ & $-(-)$ \\
\hline II & 3.1 & 3.1 & 3.1 & 3.1 & 2.9 & 2.9 & 2.9 \\
\hline Ap & 0.7 & 0.7 & 0.7 & 0.7 & 0.6 & 0.7 & 0.6 \\
\hline $\mathrm{Cc}$ & 0.2 & 0.4 & 0.2 & 1.5 & 1.4 & 0.1 & 2.8 \\
\hline
\end{tabular}

Molecular proportions Ne:Di:Hy:Ol:Q (recalculated to $100 \%$ )

\begin{tabular}{lccccccc}
$\mathrm{Ne}$ & $-(-)$ & $-(-)$ & $-(-)$ & $-(1.3)$ & $-(-)$ & $-(-)$ & $-(0.7)$ \\
$\mathrm{Di}$ & $23.8(23.8)$ & $35.0(35.0)$ & $25.8(25.8)$ & $58.7(54.8)$ & $26.2(26.2)$ & $28.9(28.9)$ & $47.0(45.3)$ \\
$\mathrm{Hy}$ & $74.6(43.4)$ & $53.1(40.2)$ & $66.4(38.1)$ & $28.2(-)$ & $39.4(21.3)$ & $66.2(33.3)$ & $31.0(-)$ \\
$\mathrm{Ol}$ & $-(32.8)$ & $-(24.8)$ & $7.8(36.1)$ & $-(43.9)$ & $34.4(52.5)$ & $4.9(37.8)$ & $22.0(54.0)$ \\
$\mathrm{Q}$ & $1.6(-)$ & $11.9(-)$ & $-(-)$ & $13.1(-)$ & $-(-)$ & $-(-)$ & $-(-)$ \\
\hline
\end{tabular}

${ }^{\text {a}}$ Values in parentheses adjusted for alteration (see text).

rocks of Core 18 , there appears to be little difference between the two groups of rocks. These slight apparent differences do not seem to be related to discernible differences in major-element chemistry or to differences in degree of alteration. Minor elements are within the ranges, and approximate the mean values, of those in olivine tholeiites generally, as reported by Prinz (1967). Element ratios (Table 4), compared to those of Engel et al. (1965), are similar to oceanic tholeiitic compositions except for ratios involving $\mathrm{K}$, which is unusually high. $\mathrm{Ba} / \mathrm{Sr}$ values are closer to those of average oceanic tholeiite than alkali basalt. 
TABLE 3

Minor-Element Content of Basalts From Site $265^{\mathrm{a}}$

\begin{tabular}{|c|c|c|c|c|c|c|c|}
\hline $\begin{array}{l}\text { Sample } \\
\text { Plot Symbol }\end{array}$ & $\begin{array}{c}17-1-2 \\
\mathrm{~A}\end{array}$ & $\begin{array}{c}17-1-8 \\
\mathrm{~B}\end{array}$ & $\begin{array}{c}17-1-21 \\
C\end{array}$ & $\underset{\mathrm{D}}{18-1-3}$ & $\begin{array}{c}18-1-6 \\
E\end{array}$ & $\begin{array}{c}18-2-9 \\
F\end{array}$ & $\begin{array}{c}18, \mathrm{CC} \\
\mathrm{G}\end{array}$ \\
\hline $\begin{array}{l}\mathrm{Ag} \\
\mathrm{Au}(\mathrm{ppb}) \\
\mathrm{B} \\
\mathrm{Ba} \\
\mathrm{Ce}\end{array}$ & $\begin{array}{l}0.05 \\
0.3 \\
\mathrm{~N} \\
85 \\
19.1\end{array}$ & $\begin{array}{c}0.02 \\
0.8 \\
\mathrm{~N} \\
100 \\
23.5\end{array}$ & $\begin{array}{c}0.02 \\
0.5 \\
\mathrm{~N} \\
100 \\
21.0\end{array}$ & $\begin{array}{l}0.03 \\
0.2 \\
70 \\
85 \\
21.0\end{array}$ & $\begin{array}{l}0.27 \\
0.3 \\
\mathrm{~N} \\
60 \\
19.5\end{array}$ & $\begin{array}{l}0.02 \\
0.2 \\
40 \\
85 \\
19.4\end{array}$ & $\begin{array}{c}0.05 \\
0.2 \\
\mathrm{~N} \\
70 \\
19.9\end{array}$ \\
\hline $\begin{array}{l}\mathrm{Co} \\
\mathrm{Cr} \\
\mathrm{Cr} * \\
\mathrm{Cu} \\
\mathrm{Eu}\end{array}$ & $\begin{array}{r}36 \\
200 \\
252 \\
65 \\
1.4\end{array}$ & $\begin{array}{r}40 \\
400 \\
266 \\
65 \\
1.3\end{array}$ & $\begin{array}{c}20 \\
370 \\
278 \\
50 \\
1.3\end{array}$ & $\begin{array}{c}38 \\
500 \\
308 \\
65 \\
1.3\end{array}$ & $\begin{array}{c}50 \\
700 \\
388 \\
65 \\
1.2\end{array}$ & $\begin{array}{c}40 \\
500 \\
273 \\
65 \\
1.3\end{array}$ & $\begin{array}{c}40 \\
700 \\
415 \\
60 \\
1.2\end{array}$ \\
\hline $\begin{array}{l}\mathrm{Ga} \\
\mathrm{Hf} \\
\mathrm{La} \\
\mathrm{Mn} \\
\mathrm{Nd}\end{array}$ & $\begin{array}{c}24 \\
2.8 \\
\mathrm{~N} \\
900 \\
16\end{array}$ & $\begin{array}{c}16 \\
2.8 \\
N \\
800 \\
19\end{array}$ & $\begin{array}{c}10 \\
2.7 \\
\mathrm{~N} \\
800 \\
16\end{array}$ & $\begin{array}{c}15 \\
2.7 \\
N \\
1000 \\
11\end{array}$ & $\begin{array}{c}15 \\
2.4 \\
\mathrm{~N} \\
900 \\
14\end{array}$ & $\begin{array}{c}15 \\
2.6 \\
N \\
700 \\
12\end{array}$ & $\begin{array}{c}15 \\
2.6 \\
N \\
850 \\
13\end{array}$ \\
\hline $\begin{array}{l}\mathrm{Ni} \\
\mathrm{Pb} \\
\mathrm{S} \\
\mathrm{Sb} \\
\mathrm{Sc}\end{array}$ & $\begin{array}{c}170 \\
\mathrm{~N} \\
200 \\
\mathrm{~N} \\
28\end{array}$ & $\begin{array}{c}170 \\
\mathrm{~N} \\
0 \\
\mathrm{~N} \\
36\end{array}$ & $\begin{array}{c}200 \\
\mathrm{~N} \\
200 \\
0.3 \\
38\end{array}$ & $\begin{array}{c}160 \\
\mathrm{~N} \\
0 \\
0.5 \\
28\end{array}$ & $\begin{array}{c}290 \\
\mathrm{~N} \\
0 \\
\mathrm{~N} \\
38\end{array}$ & $\begin{array}{c}210 \\
300 \\
\mathrm{~N} \\
36\end{array}$ & $\begin{array}{c}270 \\
\mathrm{~N} \\
0 \\
0.2 \\
28\end{array}$ \\
\hline $\begin{array}{l}\text { Sr } \\
\text { Ta } \\
\text { Th } \\
\text { Th* } \\
\text { U }\end{array}$ & $\begin{array}{l}300 \\
0.6 \\
1.6 \\
2 \\
0.28\end{array}$ & $\begin{array}{c}340 \\
0.7 \\
\mathrm{~N} \\
<1 \\
0.48\end{array}$ & $\begin{array}{c}280 \\
0.9 \\
\mathrm{~N} \\
0.6 \\
0.31\end{array}$ & $\begin{array}{c}300 \\
0.8 \\
\mathrm{~N} \\
<1 \\
0.34\end{array}$ & $\begin{array}{c}300 \\
0.7 \\
\mathrm{~N} \\
\mathrm{~N} \\
0.45\end{array}$ & $\begin{array}{c}340 \\
0.7 \\
\mathrm{~N} \\
<1 \\
0.33\end{array}$ & $\begin{array}{c}340 \\
0.8 \\
\mathrm{~N} \\
\mathrm{~N} \\
0.41\end{array}$ \\
\hline $\begin{array}{l}\mathrm{V} \\
\mathrm{Y} \\
\mathrm{Yb} \\
\mathrm{Yb} * \\
\mathrm{Zr}\end{array}$ & $\begin{array}{c}180 \\
40 \\
2 \\
3.0 \\
120\end{array}$ & $\begin{array}{c}180 \\
50 \\
2 \\
2.9 \\
110\end{array}$ & $\begin{array}{c}100 \\
50 \\
2 \\
2.8 \\
120\end{array}$ & $\begin{array}{c}200 \\
40 \\
2 \\
2.8 \\
100\end{array}$ & $\begin{array}{c}200 \\
50 \\
2 \\
2.6 \\
130\end{array}$ & $\begin{array}{c}200 \\
50 \\
2 \\
2.8 \\
130\end{array}$ & $\begin{array}{c}200 \\
30 \\
2 \\
2.9 \\
120\end{array}$ \\
\hline
\end{tabular}

${ }^{a}$ See text for analysts and methods; all values in ppm except for $\mathrm{Au}$ in ppb; N, element sought but below detectability limits.

\section{SITE 266, SOUTHEAST INDIAN RIDGE}

\section{General}

Site 266 is located on the south flank of the Southeast Indian Ridge at $56^{\circ} 24.13^{\prime} \mathrm{S}, 110^{\circ} 06.70^{\prime} \mathrm{E}$ in a water depth of 4173 meters. Basalt was encountered at a subbottom depth of 370.3 meters and was cored continuously to 384 meters. Recovery of basalt was only $14 \%$, mostly as small fragments $3-5 \mathrm{~cm}$ long and several about $10 \mathrm{~cm}$ long totaling $45 \mathrm{~cm}$ in Core 23 and $150 \mathrm{~cm}$ in Core 24 in addition to material in core catchers. Basalt fragments at the top of Core 23 are mixed with chalky and clayey drilling breccia which is probably an uphole contaminant.

Oceanic basement at the site is inferred from magnetic lineations to be 25-27 m.y. old. The sampled basalt underlies semilithified clayey nanno chalk of early Miocene age (see Site 266 paleontology report, this volume). Fission-track dating of a fragment of black, aphyric, nonhydrated glass, Sample 266-23-1-4, from near the top of the basalt unit yielded a minimum age of $17.7 \pm 4.4(\sigma)$ m.y. ${ }^{1}$ The age is considered minimum,

\footnotetext{
'Fission track analysis by C. W. Napser, U.S. Geological Survey. Analytical data: fossil track density $=445$ tracks $/ \mathrm{cm}^{2}$; induced track density $=1767$ tracks $/ \mathrm{cm}^{2} ;$ neutron dose $=1.15 \times 10^{15} \mathrm{n} / \mathrm{cm}^{2} ; U \approx$ $0.1 \mathrm{ppm} ; \lambda \mathrm{F}=6.85 \times 10^{-17} \mathrm{yr}^{-1}$.
}

because no track-annealing experiments were done on the sample, and some track fading may occur even at low sea-floor temperatures of a few degrees C (Macdougall, 1973). There is, however, no chemical or visual evidence of alteration of the glass, which has very low oxidation index and low $\mathrm{H}_{2} \mathrm{O}$ and $\mathrm{CO}_{2}$ contents (Table $5)$. The fission-track age agrees reasonably well with paleontologic dating, but is younger than crustal age inferred from magnetic lineations suggesting the possibility that the samples here represent an intrasediment basalt body.

\section{Petrography}

Except for a single sample of fresh black glass, the recovered basalts are medium to dark gray devitrified glasses containing a few percent vesicles and white, calcite-filled amygdules. No evidence of pillow structure was seen in the core, nor that more than a single cooling unit is represented. All rocks are free of phenocrysts and pseudomorphs of phenocrysts, in contrast to Site 265 glasses which typically carry microphenocrystic olivine and Site 267 glass with microphenocrystic plagioclase. The Site 266 rocks consist predominantly of variolitic, radiating masses of finely plumose, untwinned plagioclase in a turbid matrix of optically unidentifiable microcrystalline material. Amygdules and rare, thin, anastomosing veinlets are filled with calcite, smectite, translucent iron oxides, and zeolite(?). Other than the 
TABLE 4

Modes and Selected Characteristics of Site 265 Basalts

\begin{tabular}{|c|c|c|c|c|c|c|c|}
\hline $\begin{array}{l}\text { Sample } \\
\text { Plot Symbol }\end{array}$ & $\begin{array}{c}17-1-2 \\
\mathrm{~A}\end{array}$ & $\begin{array}{c}17-1-8 \\
B\end{array}$ & $\begin{array}{c}17-1-21 \\
C\end{array}$ & $\begin{array}{c}18-1-3 \\
D\end{array}$ & $\underset{\mathrm{E}}{18-1-6}$ & $\underset{F}{18-2-9}$ & $\begin{array}{c}18, \mathrm{CC} \\
\mathrm{G}\end{array}$ \\
\hline Mode & n.d. & n.d. & n.d. & & & & \\
\hline Plagioclase & & & & 39.7 & 42.6 & 41.7 & 42.0 \\
\hline Pyroxene & & & & 35.5 & 29.3 & 34.8 & 22.8 \\
\hline Olivine & & & & tr. & 13.7 & 1.7 & 6.9 \\
\hline $\begin{array}{l}\text { Opaque } \\
\text { oxide }\end{array}$ & & & & 4.2 & 3.6 & 4.9 & 1.5 \\
\hline $\begin{array}{c}\text { Alteration } \\
\text { products }\end{array}$ & & & & 16.0 & 6.2 & 7.4 & 24.8 \\
\hline Carbonate & & & & - & - & - & 1.3 \\
\hline $\begin{array}{l}\text { Vesicles and } \\
\text { amygdules }\end{array}$ & & & & 4.6 & 4.6 & 9.5 & 0.7 \\
\hline Rock Color & $\begin{array}{l}\text { Med gray, } \\
\text { N5; few } \\
\text { spots brn } \\
\text { gray } 5 \text { YR4/1 }\end{array}$ & $\begin{array}{l}\text { Same as } \\
17-1-2\end{array}$ & $\begin{array}{l}\text { Same as } \\
17-1-2\end{array}$ & $\begin{array}{r}\text { Med dk } \\
\text { gray, N4 }\end{array}$ & $\begin{array}{l}\text { Same as } \\
18-1-3\end{array}$ & $\begin{array}{l}\text { Same as } \\
18-1-3\end{array}$ & $\begin{array}{c}\text { Same as } \\
18-1-3\end{array}$ \\
\hline Density $(\mathrm{g} / \mathrm{cc})$ & 2.79 & 2.76 & 2.81 & 2.87 & 2.90 & 2.74 & 2.84 \\
\hline \multicolumn{8}{|c|}{ Plagioclase compositions } \\
\hline Normative & 58.6 & 54.8 & 53.8 & 52.5 & 56.6 & 55.2 & 51.2 \\
\hline Optical & n.d. & n.d. & n.d. & $60-70$ & $66-72$ & n.d. & $65-73$ \\
\hline \multicolumn{8}{|l|}{ Ratios } \\
\hline $\mathrm{Ba} / \mathrm{Sr}$ & 0.28 & 0.29 & 0.36 & 0.28 & 0.20 & 0.25 & 0.21 \\
\hline $\mathrm{Ni} / \mathrm{Co}$ & 4.7 & 4.3 & 10.0 & 4.2 & 5.8 & 5.3 & 6.8 \\
\hline $\mathrm{Na} / \mathrm{K}$ & 3.4 & 3.7 & 4.0 & 2.9 & 3.1 & 3.9 & 3.8 \\
\hline $\mathrm{K} / \mathrm{Zr}$ & 52 & 55 & 47 & 83 & 49 & 41 & 52 \\
\hline $\mathrm{K} / \mathrm{Ba}$ & 73 & 61 & 56 & 98 & 107 & 62 & 89 \\
\hline $\mathrm{K} / \mathrm{Cr} *$ & 25 & 23 & 20 & 27 & 16 & 19 & 15 \\
\hline Oxidation index ${ }^{a}$ & 55.6 & 64.4 & 53.5 & 71.3 & 41.2 & 62.8 & 55.6 \\
\hline $\mathrm{FeO}^{*} / \mathrm{MgO}$ & 1.2 & 1.3 & 0.9 & 2.1 & 0.8 & 0.9 & 1.1 \\
\hline Mafic index ${ }^{a}$ & 54.9 & 57.2 & 50.0 & 69.2 & 45.9 & 50.0 & 52.9 \\
\hline D. I. ${ }^{\mathrm{a}}$ & 28.6 & 31.6 & 29.8 & 34.3 & 27.6 & 28.2 & 31.9 \\
\hline
\end{tabular}

${ }^{\mathrm{a}}$ See text.

TABLE 5

Major-0xide Analyses of Basalts From Site 266, Southeast Indian Ridge

\begin{tabular}{|c|c|c|c|c|c|}
\hline $\begin{array}{c}\text { Sample } \\
\text { Depth in Core }(\mathrm{cm}) \\
\text { Plot Symbol }\end{array}$ & $\begin{array}{c}23-1-1 \\
0-10 \\
H\end{array}$ & $\begin{array}{c}23-1-4 \\
18-22 \\
\text { I }\end{array}$ & $\begin{array}{c}24-1-6 \\
29-32 \\
\mathrm{~J}\end{array}$ & $\begin{array}{c}24-1-15 \\
78-81 \\
\mathrm{~K}\end{array}$ & $\begin{array}{c}24, \mathrm{CC} \\
\mathrm{L}\end{array}$ \\
\hline $\mathrm{SiO}_{2}$ & 49.1 & 48.5 & 46.7 & 49.4 & 50.1 \\
\hline $\mathrm{A}_{2}{ }_{2} \mathrm{O}_{3}$ & 14.4 & 16.0 & 14.1 & 14.7 & 14.6 \\
\hline $\mathrm{Fe}_{2} \mathrm{O}_{3}$ & 5.7 & 1.9 & 6.9 & 5.3 & 3.4 \\
\hline $\begin{array}{l}\mathrm{FeO} \\
\mathrm{MgO} \\
\mathrm{CaO} \\
\mathrm{Na}_{2} \mathrm{O}\end{array}$ & $\begin{array}{r}5.4 \\
5.8 \\
10.1 \\
2.7\end{array}$ & $\begin{array}{l}9.3 \\
7.1 \\
9.6 \\
2.9\end{array}$ & $\begin{array}{r}4.7 \\
5.4 \\
12.0 \\
2.9\end{array}$ & $\begin{array}{l}6.1 \\
6.4 \\
9.6 \\
2.5\end{array}$ & $\begin{array}{l}8.1 \\
6.9 \\
9.8 \\
2.6\end{array}$ \\
\hline $\mathrm{K}_{2} \mathrm{O}$ & 0.73 & 0.47 & 0.57 & 0.76 & 0.36 \\
\hline $\mathrm{H}_{2} \mathrm{O}+$ & 1.3 & 0.88 & 1.2 & 1.4 & 1.1 \\
\hline $\mathrm{H}_{2} \mathrm{O}-$ & 1.9 & 0.12 & 1.7 & 1.1 & 0.52 \\
\hline $\mathrm{TiO}_{2}$ & 2.1 & 2.0 & 2.0 & 2.0 & 2.0 \\
\hline $\mathrm{P}_{2} \mathrm{O}_{5}$ & 0.29 & 0.28 & 0.30 & 0.28 & 0.29 \\
\hline $\begin{array}{l}\mathrm{MnO} \\
\mathrm{CO}_{2}\end{array}$ & $\begin{array}{l}0.22 \\
0.25\end{array}$ & $\begin{array}{l}0.23 \\
0.01\end{array}$ & $\begin{array}{l}0.23 \\
1.3\end{array}$ & $\begin{array}{l}0.20 \\
0.05\end{array}$ & $\begin{array}{l}0.22 \\
0.01\end{array}$ \\
\hline Total & 100.0 & 99.3 & 100.0 & 99.8 & 100.0 \\
\hline
\end{tabular}


comparative freshness and lack of crystallinity of Sample 266-23-1-4, there is no significant petrographic variation discernible between any of the analyzed samples of Table 5.

\section{Chemistry}

Major elements: Major-oxide analyses for five samples are given in Table 5. Of the five, the fresh aphyric glass (Sample 266-23-1-4) probably most closely approaches original magma composition. Chemical indicators of its freshness are very low oxidation index and comparatively low total $\mathrm{H}_{2} \mathrm{O}$ and $\mathrm{CO}_{2}$. The analysis of this glass falls within the range of MORB reported by Cann (1971), except for having high $\mathrm{K}_{2} \mathrm{O}$ and $\mathrm{P}_{2} \mathrm{O}_{5}$ that approximate values in Melson's (1973) type-KP basaltic glass. The slightly higher $\mathrm{Al}_{2} \mathrm{O}_{3}$ and $\mathrm{MgO}$ in the glass compared to the devitrified Site 266 glasses cannot be accounted for by plagioclase or olivine concentration because all samples are free of phenocrysts. Assuming that the fresh glass is representative of a uniform parent magma for all, variations between analyses must result from chemical transfer and oxidation during alteration and from analytical error. Alteration effects at Site 266 appear different from palagonitization described by Melson (1973) which results in loss of $\mathrm{CaO}, \mathrm{MgO}, \mathrm{P}_{2} \mathrm{O}_{5}$, and $\mathrm{Na}_{2} \mathrm{O}$, and increase of $\mathrm{K}_{2} \mathrm{O}, \mathrm{Fe}$, and $\mathrm{TiO}_{2}$.

Norms of the Site 266 basalts are given in Table 6. Except for the fresh glass, norms calculated from unadjusted analyses indicate oversaturated tholeiite compositions as they all contain Q and Hy (Yoder and Tilley, 1962); the fresh glass, containing $\mathrm{Hy}$ and $\mathrm{Ol}$, is an olivine tholeiite. Adjustment of analyses for $\mathrm{Fe}$ oxidation results in a marked decrease in the amount of $Q$ in three of the devitrified glasses and the appearance of $\mathrm{Ol}$ in the fourth. However, if all $\mathrm{Fe}_{2} \mathrm{O}_{3}$ were recalculated as $\mathrm{FeO}$, rather than only that in excess of $1.5 \%$, following Cann (1971), all norms would be free of $Q$ and would be olivine tholeiitic.

Minor elements: There appears to be little systematic difference in minor-element content between the fresh and the devitrified glass at Site 266 (Table 7). Ni/Co ratios (Table 8 ) are slightly higher than in average basalt (Fleischer, 1968), about the same as in oceanic tholeiite (Engle et al., 1965), and much lower than in Site 265 basalt. $\mathrm{Na} / \mathrm{K}, \mathrm{K} / \mathrm{Zr}$, and $\mathrm{K} / \mathrm{Cr}$ ratios more closely approach those in alkali basalt than in oceanic tholeiite, but $\mathrm{K} / \mathrm{Ba}$ of Site 266 basalt is unusually high and more like that of oceanic tholeiite. These ratios reflect the characteristically high $\mathrm{K}$ values in these rocks. On the other hand, ratios such as $\mathrm{Ba} / \mathrm{Sr}$ (Gast, 1968) and $\mathrm{Ni} / \mathrm{Co}$ are suggestive more of oceanic-tholeiite than alkali-basalt compositions.

\section{SITE 267, SOUTH INDIAN BASIN}

\section{General}

Site 267 lies in an area of low topographic relief, near the western margin of the vast abyssal plain of the South Indian Basin, at $59^{\circ} 15.74^{\prime} \mathrm{S}, 104^{\circ} 29.30^{\prime} \mathrm{E}$ in a water depth of 4564 meters. Three holes were drilled at the site. Two of these, Hole 267 and Hole 267A, were drilled at the above geographic coordinates, and the

TABLE 6

CIPW Weight Norms of Rocks From Site $266^{\mathrm{a}}$

\begin{tabular}{|c|c|c|c|c|c|}
\hline $\begin{array}{l}\text { Sample } \\
\text { Plot } \\
\text { Symbol }\end{array}$ & $23-1-1$ & $23-1-4$ & $24-1-6$ & $\begin{array}{c}24-1-15 \\
\mathrm{~K}\end{array}$ & $\begin{array}{c}24, \mathrm{CC} \\
\mathrm{L}\end{array}$ \\
\hline Q & $5.6(0.7)$ & $-(-)$ & $3.8(-)$ & $5.1(0.8)$ & $3.2(1.1)$ \\
\hline Or & 4.4 & 2.8 & 3.4 & 4.6 & 2.1 \\
\hline $\mathrm{Ab}$ & 23.3 & 24.7 & 25.0 & 21.4 & 22.1 \\
\hline An & 25.5 & 29.5 & 24.2 & 27.0 & 27.2 \\
\hline Wo & $9.2(9.4)$ & $6.9(7.0)$ & $10.9(11.1)$ & $8.0(8.1)$ & $8.2(8.3)$ \\
\hline Di $\left\{\begin{array}{l}\text { En } \\
\text { Fs }\end{array}\right.$ & $\begin{array}{l}7.1(4.9) \\
1.1(4.2)\end{array}$ & $\begin{array}{l}3.9(3.8) \\
2.8(2.9)\end{array}$ & $\begin{array}{c}9.4(5.4) \\
-(5.4)\end{array}$ & $\begin{array}{l}5.8(4.3) \\
1.4(3.6)\end{array}$ & $\begin{array}{l}5.0(4.5) \\
2.7(3.6)\end{array}$ \\
\hline Hy $\left\{\begin{array}{l}\text { En } \\
\text {. }\end{array}\right.$ & $7.6(10.1)$ & $8.1(7.4)$ & $4.3(3.9)$ & $10.3(12.2)$ & $12.2(13.0)$ \\
\hline $\begin{array}{l}\text { Fs } \\
\text { Fo }\end{array}$ & $\begin{array}{c}1.1(8.8) \\
-(-)\end{array}$ & $\begin{array}{l}5.8(5.7) \\
4.1(4.8)\end{array}$ & $\begin{array}{l}-(3.9) \\
-(3.2)\end{array}$ & $\begin{array}{c}25(10.1) \\
-(-)\end{array}$ & $\begin{array}{l}6.5(10.5) \\
-(-)\end{array}$ \\
\hline Ol $\left\{_{\mathrm{Fa}}\right.$ & $-(-)$ & $3.2(4.0)$ & $-(3.6)$ & $-(-)$ & $-(-)$ \\
\hline Mt & $8.4(2.3)$ & $2.8(2.2)$ & $10.2(2.3)$ & $7.8(2.3)$ & $5.0(2.2)$ \\
\hline Il & 4.1 & 3.8 & 3.9 & 3.8 & 3.8 \\
\hline Ap & 0.7 & 0.7 & 0.7 & 0.7 & 0.7 \\
\hline $\mathrm{Cc}$ & 0.6 & - & 3.0 & 0.1 & - \\
\hline
\end{tabular}

Molecular proportions $\mathrm{Ne}: \mathrm{Di}: \mathrm{Hy}: \mathrm{Ol}: \mathrm{Q}$ (recalculated to $100 \%$ )

\begin{tabular}{lccccc}
$\mathrm{Ne}$ & $-(-)$ & $-(-)$ & $-(-)$ & $-(-)$ & $-(-)$ \\
$\mathrm{Di}$ & $30.9(30.9)$ & $26.1(26.1)$ & $46.6(46.6)$ & $24.9(24.9)$ & $23.9(23.9)$ \\
$\mathrm{Hy}$ & $32.8(64.2)$ & $54.1(50.6)$ & $21.5(33.6)$ & $44.2(70.3)$ & $57.9(70.1)$ \\
$\mathrm{Ol}$ & $-(-)$ & $19.8(23.3)$ & $-(19.8)$ & $-(-)$ & $-(-)$ \\
$\mathrm{Q}$ & $36.3(4.9)$ & $-(-)$ & $31.9(-)$ & $30.9(4.8)$ & $18.2(6.0)$ \\
\hline
\end{tabular}

${ }^{a}$ Values in parentheses adjusted for alteration (see text). 
TABLE 7

Minor-Element Content of Basalts From Site $266^{\mathrm{a}}$

\begin{tabular}{|c|c|c|c|c|c|}
\hline $\begin{array}{l}\text { Sample } \\
\text { Plot Symbol }\end{array}$ & $\begin{array}{c}23-1-1 \\
H\end{array}$ & $\begin{array}{c}23-1-4 \\
I\end{array}$ & $\underset{\mathrm{J}}{24-1-6}$ & $\underset{\mathrm{K}}{24-1-15}$ & $\underset{\mathrm{L}}{24, \mathrm{CC}}$ \\
\hline $\begin{array}{l}\mathrm{Ag} \\
\mathrm{Au}(\mathrm{ppb}) \\
\mathrm{B} \\
\mathrm{Ba} \\
\mathrm{Ce}\end{array}$ & $\begin{array}{c}0.18 \\
0.8 \\
50 \\
100 \\
18.6\end{array}$ & $\begin{array}{c}0.07,0.03 \\
0.3,0.3 \\
\mathrm{~N} \\
28 \\
19.1\end{array}$ & $\begin{array}{l}0.01 \\
0.3 \\
50 \\
24 \\
19.9\end{array}$ & $\begin{array}{l}0.18 \\
0.3 \\
55 \\
24 \\
19.5\end{array}$ & $\begin{array}{l}0.02 \\
0.2 \\
\mathrm{~N} \\
26 \\
18.5\end{array}$ \\
\hline $\begin{array}{l}\mathrm{Co} \\
\mathrm{Cr} \\
\mathrm{Cr} * \\
\mathrm{Cu} \\
\mathrm{Eu}\end{array}$ & $\begin{array}{c}40 \\
280 \\
149 \\
80 \\
1.4\end{array}$ & $\begin{array}{r}40 \\
250 \\
158 \\
55 \\
1.6\end{array}$ & $\begin{array}{c}40 \\
300 \\
146 \\
60 \\
1.6\end{array}$ & $\begin{array}{c}36 \\
250 \\
143 \\
55 \\
1.6\end{array}$ & $\begin{array}{c}40 \\
250 \\
157 \\
70 \\
1.4\end{array}$ \\
\hline $\begin{array}{l}\mathrm{Ga} \\
\mathrm{Hf} \\
\mathrm{La} \\
\mathrm{Mn} \\
\mathrm{Nd}\end{array}$ & $\begin{array}{c}20 \\
3.6 \\
\mathrm{~N} \\
1200 \\
19\end{array}$ & $\begin{array}{c}20 \\
3.9 \\
\mathrm{~N} \\
1000 \\
11\end{array}$ & $\begin{array}{c}18 \\
4.0 \\
N \\
1200 \\
15\end{array}$ & $\begin{array}{c}18 \\
3.6 \\
N \\
1200 \\
15\end{array}$ & $\begin{array}{c}16 \\
2.8 \\
\mathrm{~N} \\
1300 \\
20\end{array}$ \\
\hline $\begin{array}{l}\mathrm{Ni} \\
\mathrm{Pb} \\
\mathrm{S} \\
\mathrm{Sb} \\
\mathrm{Sc}\end{array}$ & $\begin{array}{r}130 \\
\mathrm{~N} \\
300 \\
\mathrm{~N} \\
38\end{array}$ & $\begin{array}{r}110 \\
N \\
100 \\
N \\
40\end{array}$ & $\begin{array}{c}130 \\
\mathrm{~N} \\
0 \\
\mathrm{~N} \\
38\end{array}$ & $\begin{array}{c}110 \\
\mathrm{~N} \\
600 \\
0.5 \\
48\end{array}$ & $\begin{array}{c}130 \\
\mathrm{~N} \\
400 \\
0.3 \\
40\end{array}$ \\
\hline $\begin{array}{l}\mathrm{Sr} \\
\mathrm{Ta} \\
\mathrm{Th} \\
\mathrm{Th} * \\
\mathrm{U}\end{array}$ & $\begin{array}{c}200 \\
0.4 \\
\mathrm{~N} \\
<1 \\
0.27\end{array}$ & $\begin{array}{c}130 \\
0.4 \\
-\quad 1 \\
-\end{array}$ & $\begin{array}{l}190 \\
0.4 \\
\mathrm{~N} \\
\mathrm{~N} \\
0.37\end{array}$ & $\begin{array}{l}130 \\
0.4 \\
\mathrm{~N} \\
\mathrm{~N} \\
0.29\end{array}$ & $\begin{array}{l}130 \\
0.4 \\
\mathrm{~N} \\
\mathrm{~N} \\
0.18\end{array}$ \\
\hline $\begin{array}{l}\mathrm{V} \\
\mathrm{Y} \\
\mathrm{Yb} \\
\mathrm{Yb} * \\
\mathrm{Zr}\end{array}$ & $\begin{array}{c}280 \\
60 \\
5 \\
5.5 \\
160\end{array}$ & $\begin{array}{r}270 \\
40 \\
5 \\
5.6 \\
160\end{array}$ & $\begin{array}{c}360 \\
60 \\
5 \\
5.4 \\
150\end{array}$ & $\begin{array}{c}320 \\
60 \\
5 \\
5.1 \\
150\end{array}$ & $\begin{array}{c}350 \\
60 \\
5 \\
5.6 \\
150\end{array}$ \\
\hline
\end{tabular}

${ }^{a}$ See text for analysts and methods; all values in ppm except for $\mathrm{Au}$ in ppb; $\mathrm{N}$, element sought but below detectability limits.

TABLE 8

Selected Characteristics of Site 266 Basalts

\begin{tabular}{|c|c|c|c|c|c|}
\hline $\begin{array}{l}\text { Sample } \\
\text { Plot Symbol }\end{array}$ & $\begin{array}{c}23-1-1 \\
\mathrm{H}\end{array}$ & $\begin{array}{c}23-1-4 \\
\text { I }\end{array}$ & $\begin{array}{c}24-1-6 \\
J\end{array}$ & $\begin{array}{c}24-1-15 \\
K\end{array}$ & $\underset{\mathrm{L}}{24, \mathrm{CC}}$ \\
\hline Rock color & $\begin{array}{r}\text { Med dk } \\
\text { gray, N4 }\end{array}$ & $\begin{array}{l}\text { Black, } \\
\text { N1 }\end{array}$ & $\begin{array}{l}\text { Med gray, } \\
\text { N5 }\end{array}$ & $\begin{array}{r}\text { Med dk } \\
\text { gray, N4 }\end{array}$ & $\begin{array}{c}\text { Dk gray, } \\
\text { N3 }\end{array}$ \\
\hline Density $(\mathrm{g} / \mathrm{cc})$ & 2.81 & 2.77 & 2.68 & 2.90 & 2.91 \\
\hline \multicolumn{6}{|c|}{ Plagioclase compositions } \\
\hline Normative & 52.3 & 54.4 & 49.2 & 55.7 & 55.2 \\
\hline \multicolumn{6}{|l|}{ Ratios } \\
\hline $\mathrm{Ba} / \mathrm{Sr}$ & 0.50 & 0.22 & 0.13 & 0.18 & 0.20 \\
\hline $\mathrm{Ni} / \mathrm{Co}$ & 3.3 & 2.8 & 3.3 & 3.1 & 3.3 \\
\hline $\mathrm{Na} / \mathrm{K}$ & 3.3 & 5.5 & 4.6 & 2.9 & 6.4 \\
\hline $\mathrm{K} / \mathrm{Zr}$ & 38 & 24 & 31 & 42 & 20 \\
\hline $\mathrm{K} / \mathrm{Ba}$ & 61 & 139 & 196 & 263 & 115 \\
\hline $\mathrm{K} / \mathrm{Cr} *$ & 41 & 25 & 32 & 44 & 19 \\
\hline Oxidation index ${ }^{a}$ & 51.4 & 17.0 & 59.5 & 46.5 & 29.6 \\
\hline $\mathrm{FeO} * / \mathrm{MgO}$ & 1.8 & 1.6 & 2.0 & 1.7 & 1.6 \\
\hline Mafic index ${ }^{a}$ & 65.7 & 61.2 & 68.2 & 64.0 & 62.5 \\
\hline D.I. ${ }^{\mathrm{a}}$ & 33.3 & 27.5 & 32.2 & 31.1 & 27.5 \\
\hline
\end{tabular}

${ }^{\text {a See text. }}$ 
third, Hole $267 \mathrm{~B}$, was drilled $2.6 \mathrm{~km}$ away at $59^{\circ} 14.55^{\prime} \mathrm{S}$, $104^{\circ} 29.94^{\prime} \mathrm{E}$ in a water depth of 4539 meters. At Hole 267 basalt was reached at a subbottom depth of about 205 meters and cored to 219.5 meters at which depth drilling terminated. Basalt recovery was only $16 \%$, and mostly consists of small fragments $3-5 \mathrm{~cm}$ long totaling $80 \mathrm{~cm}$ in Core 6 , and fragments $3-10 \mathrm{~cm}$ long totaling $150 \mathrm{~cm}$ in Core 7; additional material was recovered in core catchers. Basalt was not reached in Hole 267A. In Hole 267B basalt was reached at 314 meters and cored to 323 meters, with recovery of $25 \mathrm{~cm}$ of core of devitrified glassy basalt. Samples for the present study were collected only from Hole 267.

Oceanic basement at Site 267 is inferred from magnetic lineations to be about 38-45 m.y. old, but the dating is uncertain because magnetic anomalies are not well mapped in this region. The sampled basalt underlies foram-bearing micritic limestone and nanno chalk dated paleontologically as near the Eocene-Oligocene boundary. Limestone immediately above basalt, though comparatively well lithified, shows no microscopic evidence of recrystallization. A small fragment of limestone recovered from about $10 \mathrm{~cm}$ below the basalt contact is probably an uphole contaminant rather than being a stopped block in an intrusive body of basalt.

\section{Petrography}

The Site 267 basalts show little petrographic variation other than generally minor differences in crystallinity. Most are medium- to dark-gray devitrified glasses of fairly homogeneous appearance except for local mottling caused by variole structures, and the presence of a few calcite, smectite, and zeolite veinlets up to about 5 $\mathrm{mm}$ width. A few core fragments show thin selvages of darker glass. Plagioclase (labradorite) is the only phenocrystic phase in these rocks, and forms blocky, little-zoned microphenocrysts comprising several percent, but locally as much as $10 \%$, of the mode. The microphenocrysts in places form cumulophyric clusters along with minor and much smaller, poorly formed clinopyroxenes, probably augite. Vesicles and calcite-, smectite-, and zeolite-filled amygdules form up.to about $5 \%$ of the rock.

Most rocks, including analyzed Samples 267-6, CC-5 and 267-6-1-11, are devitrified glass showing plagioclase microlites in a turbid, brownish, microcrystalline and microspherulitic groundmass, in addition to the microphenocrysts described above. The microlites commonly exhibit quench textures. Interspersed with the glassy rocks are a few nearly holocrystalline rocks that are very fine grained, such as analyzed Sample 267-6-125 . The fine grain size precludes modal analysis, but the rocks appear to contain subequal amounts of needleshaped to blocky, little-zoned plagioclase in a matrix of finely granular clinopyroxene and alteration products. The intermixing of glassy and crystalline rocks suggests the presence of pillows but, as at Site 265, transitions from rim to center of these inferred structures were not recovered in any single length of core. There is no conclusive evidence at Site 267 that more than a single flow of basalt was penetrated.

\section{Chemistry}

Major elements: Table 9 shows major-oxide analyses for three samples from the upper part of the basalt. Owing to the more widespread presence of veinlets in Core 7 than in Core 6 basalt, only the latter was sampled for analysis. Sample 267-6, CC-5, having an oxidation index of 36.7 , which is unusually low for Leg 28 basalt, is apparently the least altered of the three and probably most nearly approaches original magma composition at this site. $\mathrm{K}_{2} \mathrm{O}$ and $\mathrm{P}_{2} \mathrm{O}$ values in this sample are, also, unusually low for Leg 28 basalt. Except for very slight differences in $\mathrm{Na}_{2} \mathrm{O}$ and $\mathrm{K}_{2} \mathrm{O}$, the analysis falls within the range of MORB given by Cann (1971). Alteration at this site apparently resulted in an appreciable increase only in $\mathrm{K}_{2} \mathrm{O}$ but, surprisingly, not in $\mathrm{H}_{2} \mathrm{O}$. The only other consistent changes related to oxidation appear to be a very slight loss of $\mathrm{MnO}$ and gain of $\mathrm{P}_{2} \mathrm{O}_{5}$. The slight apparent gain of $\mathrm{P}_{2} \mathrm{O}_{5}$ is surprising in view of its marked loss in glass alteration reported by Melson (1973). As at Site 266, there appears to be little consistent change of $\mathrm{Na}_{2} \mathrm{O}, \mathrm{CaO}, \mathrm{MgO}$, and $\mathrm{TiO}$ by alteration, in contrast to changes reported by Melson (1973).

Site 267 basalt norms are given in Table 10. Norms calculated from unadjusted analyses are indicative of silica-oversaturated tholeiite compositions for all rocks, and the small amounts of $\mathrm{Q}$ remaining after adjustment for $\mathrm{Fe}$ oxidation suggests original slightly oversaturated composition. Normative plagioclase compositions are more calcic than at other southeast Indian Ocean sites.

Minor elements: Values of most minor elements (Table 11) much more nearly approximate those of oceanic tholeiite than alkali basalt (Engel et al., 1965). Other than an increase in $\mathrm{Cu}$ there appears to be no systematic change in minor-element content related to increasing alteration. Ba values are unusually low, compared to other southeast Indian Ocean sites, and result in unusually high $\mathrm{K} / \mathrm{Ba}$ ratios.

TABLE 9

Major-Oxide Analyses of Basalts From Site 267, South Indian Basin

\begin{tabular}{|c|c|c|c|c|}
\hline Depth & $\begin{array}{l}\text { Sample } \\
\text { in Core }(\mathrm{cm}) \\
\text { Plot Symbol }\end{array}$ & $\begin{array}{c}6-1-11 \\
0-6 \\
M\end{array}$ & $\begin{array}{c}6-1-25 \\
57-61 \\
\mathrm{~N}\end{array}$ & $\begin{array}{c}6, \mathrm{CC}-5 \\
0\end{array}$ \\
\hline & $\mathrm{SiO}_{2}$ & 50.1 & 50.8 & 50.5 \\
\hline & $\mathrm{Al}_{2} \mathrm{O}_{3}$ & 15.4 & 15.3 & 15.4 \\
\hline & $\mathrm{Fe}_{2} \mathrm{O}_{3}$ & 4.3 & 3.8 & 3.3 \\
\hline & $\mathrm{FeO}$ & 5.3 & 5.2 & 5.7 \\
\hline & $\mathrm{MgO}$ & 7.1 & 7.4 & 7.4 \\
\hline & $\mathrm{CaO}$ & 11.7 & 11.6 & 11.9 \\
\hline & $\mathrm{Na}_{2} \mathrm{O}$ & 2.3 & 2.2 & 2.3 \\
\hline & $\mathrm{K}_{2} \mathrm{O}$ & 0.54 & 0.40 & 0.36 \\
\hline & $\mathrm{H}_{2} \mathrm{O}+$ & 0.87 & 0.89 & 0.84 \\
\hline & $\mathrm{H}_{2} \mathrm{O}-$ & 0.63 & 0.61 & 0.56 \\
\hline & $\mathrm{TiO}_{2}$ & 1.3 & 1.3 & 1.3 \\
\hline & $\mathrm{P}_{2} \mathrm{O}_{5}$ & 0.18 & 0.16 & 0.15 \\
\hline & $\mathrm{MnO}$ & 0.18 & 0.20 & 0.21 \\
\hline & $\mathrm{CO}_{2}$ & 0.02 & 0.01 & 0.01 \\
\hline & Total & 99.9 & 99.9 & 99.9 \\
\hline
\end{tabular}


TABLE 10

CIPW Weight Norms of Rocks From Site $267^{\mathrm{a}}$

\begin{tabular}{|c|c|c|c|}
\hline $\begin{array}{l}\text { Sample } \\
\text { Plot } \\
\text { Symbol }\end{array}$ & $\begin{array}{c}6-1-11 \\
\mathrm{M}\end{array}$ & $\begin{array}{c}6-1-25 \\
\mathrm{~N}\end{array}$ & $\begin{array}{c}6, \mathrm{CC}-5 \\
\mathrm{O}\end{array}$ \\
\hline Q & $3.4(0.2)$ & $4.5(1.9)$ & $2.9(0.8)$ \\
\hline Or & 3.2 & 2.4 & 2.1 \\
\hline $\mathrm{Ab}$ & 19.6 & 18.8 & 19.6 \\
\hline An & $\begin{array}{l}30.3 \\
11.2(11.3)\end{array}$ & $\begin{array}{l}30.9 \\
10.8(11.0)\end{array}$ & $\begin{array}{l}30.8 \\
11.5(11.6)\end{array}$ \\
\hline Di $\left\{\begin{array}{l}\text { En } \\
\text { Fs }\end{array}\right.$ & $\begin{array}{l}8.2(6.6) \\
2.0(4.2) \\
9.7(11.4)\end{array}$ & $\begin{array}{c}7.9(6.6) \\
2.0(3.7) \\
10.7(12.2)\end{array}$ & $\begin{array}{c}8.0(7.0) \\
2.6(4.0)\end{array}$ \\
\hline Hy $\left\{\begin{array}{l}\text { En } \\
\end{array}\right.$ & $9.7(11.4)$ & $10.7(12.2)$ & $10.6(11.7)$ \\
\hline $\begin{array}{l}\text { Mt Fs } \\
\text { Mt } \\
\text { II } \\
\text { Ap } \\
\text { Cc }\end{array}$ & $\begin{array}{l}2.4(7.3) \\
6.3 \\
2.5 \\
0.4 \\
0.0\end{array}$ & $\begin{array}{l}2.7(6.9) \\
5.6 \\
2.5 \\
0.4 \\
0.0\end{array}$ & $\begin{array}{l}3.4(6.6) \\
4.8 \\
2.5 \\
0.4 \\
0.0\end{array}$ \\
\hline
\end{tabular}

Molecular proportions $\mathrm{Ne}: \mathrm{Di}: \mathrm{Hy}: \mathrm{Ol}: \mathrm{Q}$ (recalculated to $100 \%$ )

\begin{tabular}{lccc}
$\mathrm{Ne}$ & - & - & - \\
$\mathrm{Di}$ & $36.2(36.2)$ & $31.5(31.5)$ & $35.6(35.6)$ \\
$\mathrm{Hy}$ & $42.9(62.7)$ & $42.9(57.8)$ & $47.2(59.4)$ \\
$\mathrm{Ol}$ & - & - & - \\
$\mathrm{Q}$ & $20.9(1.1)$ & $25.6(10.7)$ & $17.2(5.0)$ \\
\hline
\end{tabular}

${ }^{a}$ Values in parentheses adjusted for alteration (see text).

\section{SITE 274, BALLENY BASIN}

\section{General}

Site 274 is located near the Balleny Basin on the lower continental rise of Antarctica at $68^{\circ} 59.81^{\prime} \mathrm{S}, 173^{\circ}$ $25.64^{\prime} \mathrm{E}$ in a water depth of 3326 meters. Basalt was encountered at a subbottom depth of 408.5 meters and was cored continuously to 421 meters. Basalt recovered totals 1.7 meters in Core 44 and 1.65 meters in Core 45 for a recovery of $34 \%$ in addition to core-catcher material.

The crustal age at this site is undated by magnetic lineations but is inferred from regional studies of Weissel and Hayes (1972) to be at least as old as Eocene. The sampled basalt is overlain by silty claystone and chert of Eocene and Cretaceous(?) age (see Site 274 paleontology report, this volume). The contact between basalt and overlying sedimentary rock was not recovered.

\section{Petrography}

The general character of Site 274 basalt differs greatly from that of the southeast Indian Ocean basalts. Although quench textures are very locally shown by plagioclase and clinopyroxene, the basalts are nonspherulitic and show no evidence that glass was a significant original component. They are holocrystalline, commonly with intergranular to diabasic texture. Most rocks appear altered to some degree as indicated by the common presence of groundmass calcite, smectite, chlorite, zeolite, and brownish, translucent hydrous iron oxides. The freshest-appearing rocks are medium dark gray to medium gray in color, but the more common
TABLE 11

Minor-Element Content of Basalts From Site $267^{\mathrm{a}}$

\begin{tabular}{|c|c|c|c|}
\hline $\begin{array}{l}\text { Sample } \\
\text { Plot Symbol }\end{array}$ & $\begin{array}{c}6-1-11 \\
M\end{array}$ & $\begin{array}{c}6-1-25 \\
N\end{array}$ & $\begin{array}{c}6, \mathrm{CC}-5 \\
\mathrm{O}\end{array}$ \\
\hline $\mathrm{Ag}$ & 0.06 & 0.07 & 0.04 \\
\hline $\mathrm{Au}(\mathrm{ppb})$ & 0.3 & 0.1 & 0.23 \\
\hline B & 55 & $\mathrm{~N}$ & $\mathrm{~N}$ \\
\hline $\mathrm{Ba}$ & 9 & 5 & 16 \\
\hline $\mathrm{Ce}$ & 7 & 10 & 9 \\
\hline Co & 40 & 42 & 40 \\
\hline $\mathrm{Cr}$ & 330 & 380 & 300 \\
\hline $\mathrm{Cr} *$ & 157 & 194 & 172 \\
\hline $\mathrm{Cu}$ & 65 & 60 & 55 \\
\hline $\mathrm{Eu}$ & 0.8 & 1.0 & 1.0 \\
\hline $\mathrm{Ga}$ & 15 & 14 & 22 \\
\hline $\mathrm{Hf}$ & 1.8 & 2.2 & 2.4 \\
\hline La & $\mathrm{N}$ & $\mathrm{N}$ & $\mathrm{N}$ \\
\hline Mn & 1000 & 950 & 1200 \\
\hline $\mathrm{Nd}$ & 14 & 10 & 7 \\
\hline $\mathrm{Ni}$ & 80 & 95 & 70 \\
\hline $\mathrm{Pb}$ & $\mathrm{N}$ & $\mathrm{N}$ & $\mathrm{N}$ \\
\hline $\mathrm{S}$ & 0 & 0 & 0 \\
\hline $\mathrm{Sb}$ & 0.3 & $\mathrm{~N}$ & 0.3 \\
\hline $\mathrm{Sc}$ & 50 & 42 & 42 \\
\hline $\mathrm{Sr}$ & 190 & 200 & 190 \\
\hline $\mathrm{Ta}$ & 0.2 & 0.2 & 0.2 \\
\hline Th & $\mathrm{N}$ & $\mathrm{N}$ & $\mathrm{N}$ \\
\hline Th* & $\mathrm{N}$ & $\mathrm{N}$ & $\mathrm{N}$ \\
\hline $\mathrm{U}$ & 0.33 & 0.55 & $\mathrm{~N}$ \\
\hline V & 300 & 280 & 280 \\
\hline $\mathrm{Y}$ & 40 & 40 & 40 \\
\hline $\mathrm{Yb}$ & 3 & 3 & 3 \\
\hline $\mathrm{Yb}^{*}$ & 3.2 & 3.5 & 3.2 \\
\hline $\mathrm{Zr}$ & 80 & 75 & 75 \\
\hline
\end{tabular}

${ }^{\mathrm{a}}$ See text for analysts and methods; all values in ppm except for $\mathrm{Au}$ in ppb; N, element sought but below detectability limits.

altered ones range from brownish-gray to olive-gray or dark greenish-gray. Unusually low densities, from about 2.5 to $2.7 \mathrm{~g} / \mathrm{cc}$ (Table 16), reflect the high degree of alteration. Even the freshest-appearing analyzed basalt, Sample 274-44-1-2, has lower than average density, 2.78 $\mathrm{g} / \mathrm{cc}$, for Leg 28 basalt.

Poor core recovery makes interpretation of the occurrence uncertain. Many core fragments consist entirely of uniform-appearing basalt, except for being cut by thin veinlets. Other core fragments, however, are of altered breccia-like rock consisting of unsorted, fine to coarse, angular blocks in a fine, altered tuffaceous(?) matrix (Figure 10). The occurrences of breccia are scattered through the entire basalt unit. The entire cored unit is therefore interpreted as being breccia, probably a flow breccia. Core fragments of uniform basalt are accordingly inferred to represent large blocks in the breccia deposit rather than separate flow units or parts of homogeneous flows. The lack of glass even at the very top of the unit suggests that the lava was crystallized, or very nearly so, at the time of emplacement.

Plagioclase is the only phenocrystic phase present, commonly forming blocky, cumulophyric, microphenocrystic clusters 1-3 mm in size. Analyzed Sample 
TABLE 12

Selected Characteristics of Site 267 Basalts

\begin{tabular}{lccc}
\hline $\begin{array}{l}\text { Sample } \\
\text { Plot Symbol }\end{array}$ & $\begin{array}{c}6-1-11 \\
\mathrm{M}\end{array}$ & $\begin{array}{c}6-1-25 \\
\mathrm{~N}\end{array}$ & $\begin{array}{c}6, \mathrm{CC}-5 \\
\mathrm{O}\end{array}$ \\
\hline Rock Color & $\begin{array}{c}\text { Med gray } \\
\text { N5 }\end{array}$ & $\begin{array}{c}\text { Med gray, } \\
\text { N5 }\end{array}$ & $\begin{array}{c}\text { Med gray, } \\
\text { N5 }\end{array}$ \\
& & & \\
Density (g/cc) & 2.94 & 2.89 & 2.97 \\
& & & \\
Plagioclase Compositions & & & \\
Normative & 60.7 & 62.2 & 61.1 \\
& & & \\
Ratios & & & \\
Ba/Sr & 0.05 & 0.03 & 0.08 \\
Ni/Co & 2.0 & 2.3 & 1.8 \\
Na/K & 3.8 & 4.9 & 5.7 \\
K/Zr & 56 & 44 & 40 \\
K/Ba & 498 & 664 & 187 \\
K/Cr* & 28 & 17 & 17 \\
& & & \\
Oxidation index & 44.8 & 42.2 & 36.7 \\
FeO*/MgO & 1.3 & 1.2 & 1.2 \\
Mafic index & 57.5 & 54.9 & 54.9 \\
D.I. ${ }^{\mathrm{a}}$ & 26.2 & 25.9 & 24.6 \\
\hline
\end{tabular}

${ }^{\text {a }}$ See text.

274-44-1-2 is formed of weakly zoned labradorite microphenocrysts, constituting from several to about 10 modal percent, set in a fine intergranular-textured matrix of microlitic plagioclase, clinopyroxene, and alteration products. A few rocks, such as analyzed Samples 274-44-2-18 and 274-44, CC, show unusually coarse grain size with intergranular textures approaching diabasic size; interstices of lath-shaped labradorite are filled mainly with fine clinopyroxene, and smectite, minor calcite, and other secondary minerals. Some rocks, such as analyzed Sample 274-2-35, are highly altered dense rocks with albitized plagioclase and abundant chlorite, smectite, calcite, and zeolite. Many rocks contain several to as much as about $10 \%$ amygdules that are commonly filled with radiating zeolite or calcite. Veinlets of secondary minerals are common (Figure 8).

\section{Chemistry}

Major elements: The widespread and locally high degree of alteration of these rocks makes interpretation of chemistry uncertain. Two chemically analyzed basalts, Samples 274-45-2-35 and 274-45-2-39 (Table 13), are highly altered as indicated by their petrography, and by their unusually high oxidation indexes and high $\mathrm{H}_{2} \mathrm{O}$ and $\mathrm{K}_{2} \mathrm{O}$ contents. Two basalts, Samples 274-44-12 and 274-44-2-15, although having moderate $\mathrm{H}_{2} \mathrm{O}$ content, are comparatively little weathered as indicated by low oxidation index and probably by unusually low $\mathrm{K}_{2} \mathrm{O}$ value. The high $\mathrm{CO}_{2}$ and higher than average $\mathrm{CaO}$ probably results from microscopic carbonate veinlets seen in thin section. If it is assumed that all rocks at this site crystallized from one homogeneous magma and chemical differences are due only to changes caused by alteration, Samples 274-44-1-2 and 274-44-2-15 probably most nearly represent original magma type at Site 274. Except for unusually low $\mathrm{MgO}$ and slightly low $\mathrm{FeO}^{*}$ and $\mathrm{CaO}$ the two analyses fall within the range of
MORB of Cann (1971) and do not correspond closely with any of the other oceanic basalt types-KP, FETI, and CAMG-of Melson (1973). Except for low MgO, they correspond closely to oceanic tholeiite (Engel et al., 1965). If chemical differences at this site have resulted mainly from alteration, the apparent increase in $\mathrm{P}_{2} \mathrm{O}_{5}$ in the most-altered rocks like that at Site 267 is surprising in light of results reported by Melson (1973).

Norms of unadjusted analyses (Table 14) contain 5\%$9 \% \mathrm{Q}$, and all contain $\mathrm{Q}$ after adjusting compositions for $\mathrm{Fe}$ oxidation. At least minor amounts of $\mathrm{Q}$ remain in most rocks even if all $\mathrm{Fe}_{2} \mathrm{O}_{3}$ were recalculated as $\mathrm{FeO}$, and so the rocks are clearly of $\mathrm{SiO}_{2}$-oversaturated tholeiitic composition.

Minor elements: Minor elements (Table 15) much more closely correspond to average oceanic tholeiite than alkali basalt of Engel et al. (1965). U abundance shows poor correlation with oxidation index in contrast to results reported by Aumento (1971) that show an increase in $U$ content in alteration of Mid-Atlantic Ridge tholeiite. If alteration is the chief cause of minor-element variation, it appears to have been accompanied by increase of $\mathrm{B}, \mathrm{Ba}, \mathrm{Ce}, \mathrm{Sr}, \mathrm{Ta}$, and $\mathrm{Zr}$, and decrease of $\mathrm{Ag}$, $\mathrm{Co}, \mathrm{Ni}, \mathrm{S}$, and $\mathrm{V}$.

\section{COMPARISON OF LEG 28 BASALTS}

Basaltic rocks sampled on Leg 28 show significant petrographic and chemical diversity between sites, especially between southeast Indian Ocean sites and Site 274 near Balleny Basin. Other than differences in crystallinity and degree of alteration, the chief petrographic differences apparent are presence of microphenocrystic olivine at Site 265, contrasting with lack of olivine at all other sites, and comparative abundance of phenocrystic plagioclase at Site 274 .

Average chemical analyses for each site are compared in Table 17. Site 274 basalts are not included in the overall average for southeast Indian Ocean samples because they apparently are from a different oceanic and tectonic province. The average compositions for southeast Indian Ocean sites show progressively increasing $\mathrm{SiO}_{2}$ and decreasing $\mathrm{Na}_{2} \mathrm{O}$ and $\mathrm{K}_{2} \mathrm{O}$ in a direction southward away from the ridge axis. The $\mathrm{K}_{2} \mathrm{O}$ decrease is surprising in view of the typically low-K characteristics of ridge-axis basalt (Kay et al., 1970) compared to ridge-flank basalt. Average normative compositions (Table 18) show southward-increasing $\mathrm{SiO}_{2}$ saturation and decreasing $\mathrm{Ab}$ and Or. After norm recalculations adjusted for $\mathrm{Fe}$ oxidation, only negligible Q remains in Site 267 basalt and in Site 266 basalt original Q is replaced by minor Ol. Site 274 basalt, on the other hand, retains appreciable $Q$ even after adjusting for oxidation. Using criteria of Yoder and Tilley (1962), original Site 265 compositions were olivine tholeiite, and Sites 266 and 267 compositions were, respectively, slightly undersaturated olivine tholeiite and slightly oversaturated quartz tholeiite. Site 274 basalts were comparatively highly oversaturated quartz tholeiitic.

Much of the chemical diversity of Leg 28 basalts apparently results from deep-sea weathering as is indicated by generally high volatile content and oxidation index. 
TABLE 13

Major-Oxide Analyses of Basalts From Site 274, Balleny Basin

\begin{tabular}{lcccccc}
\hline $\begin{array}{l}\text { Sample } \\
\text { Depth }\end{array}$ & $44-1-2$ & $44-2-15$ & $44-2-18$ & $44, \mathrm{CC}$ & $45-2-35$ & $45-2-39$ \\
in Core $(\mathrm{cm})$ & $3-5$ & $139-142$ & $159-168$ & & $133-136$ & $153-155$ \\
Plot Symbol & $\mathrm{P}$ & $\mathrm{Q}$ & $\mathrm{R}$ & $\mathrm{S}$ & $\mathrm{T}$ & $\mathrm{U}$ \\
\hline $\mathrm{SiO}_{2}$ & 48.2 & 47.9 & 49.3 & 49.5 & 48.5 & 47.1 \\
$\mathrm{Al}_{2} \mathrm{O}_{3}$ & 16.8 & 16.8 & 17.1 & 17.7 & 15.6 & 15.5 \\
$\mathrm{Fe}_{2} \mathrm{O}_{3}$ & 3.0 & 3.2 & 3.7 & 3.8 & 7.3 & 6.1 \\
$\mathrm{FeO}_{\mathrm{MgO}}$ & 6.2 & 4.6 & 3.9 & 3.5 & 2.8 & 3.0 \\
$\mathrm{CaO}$ & 4.2 & 5.3 & 5.7 & 5.6 & 6.0 & 6.2 \\
$\mathrm{Na}_{2} \mathrm{O}$ & 10.7 & 10.4 & 9.6 & 9.7 & 6.6 & 7.0 \\
$\mathrm{~K}_{2} \mathrm{O}$ & 2.7 & 2.7 & 3.0 & 2.9 & 2.9 & 2.9 \\
$\mathrm{H}_{2} \mathrm{O}+$ & 0.27 & 0.21 & 0.37 & 0.42 & 1.5 & 1.3 \\
$\mathrm{H}_{2} \mathrm{O}-$ & 1.2 & 1.7 & 1.6 & 1.6 & 2.2 & 2.7 \\
$\mathrm{TiO}_{2}$ & 2.2 & 3.0 & 3.4 & 3.3 & 4.2 & 4.1 \\
$\mathrm{P}_{2} \mathrm{O}_{5}$ & 1.6 & 1.6 & 1.6 & 1.6 & 1.8 & 1.8 \\
$\mathrm{MnO}_{\mathrm{CO}}$ & 0.18 & 0.18 & 0.19 & 0.17 & 0.41 & 0.34 \\
$\mathrm{Total}_{2}$ & 0.21 & 0.15 & 0.12 & 0.09 & 0.13 & 0.19 \\
\hline & 2.6 & 2.0 & 0.35 & 0.07 & 0.08 & 2.4 \\
\hline & 100.0 & 99.7 & 99.9 & 100.0 & 100.0 & 100.8 \\
\hline
\end{tabular}

TABLE 14

CIPW Weight Norms of Analyzed Rocks From Site $274^{\mathrm{a}}$

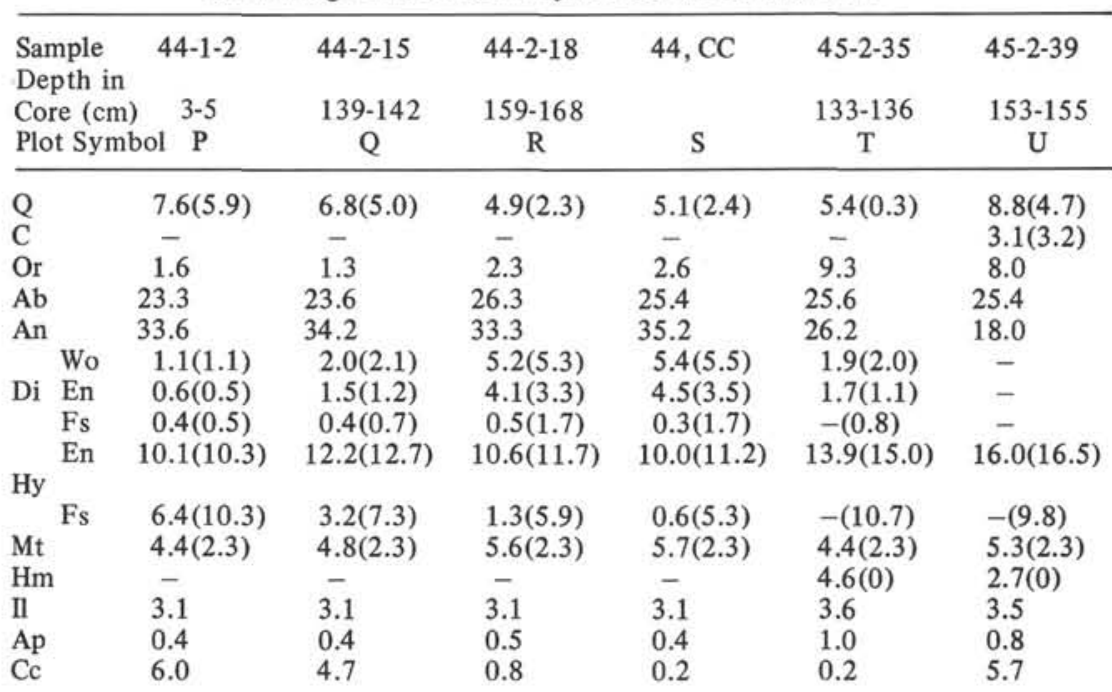

Molecular proportions Ne:Di:Hy:Ol:Q (recalculated to $100 \%$ )

\begin{tabular}{lcccccc}
$\mathrm{Ne}$ & - & - & - & - & - & - \\
$\mathrm{Di}$ & $3.3(3.3)$ & $6.3(6.3)$ & $18.7(18.7)$ & $19.9(19.9)$ & $6.8(6.8)$ & - \\
$\mathrm{Hy}$ & $52.4(62.8)$ & $52.6(64.3)$ & $47.7(65.6)$ & $44.1(63.4)$ & $56.7(91.3)$ & $52.0(75.5)$ \\
$\mathrm{Ol}$ & - & - & - & - & - & - \\
$\mathrm{Q}$ & $44.3(33.9)$ & $41.1(29.4)$ & $33.6(15.7)$ & $36.0(16.7)$ & $36.5(1.8)$ & $48.0(24.5)$ \\
\hline
\end{tabular}

${ }^{\mathrm{a}}$ Values in parentheses adjusted for alteration (see text).

Many of the basalts contain greater than $1.0 \% \mathrm{H}_{2} \mathrm{O}^{+}$, compared to an estimated $0.25 \%$ for fresh K-poor oceanic tholeiite, $0.5 \%$ for Hawaiian tholeiite of intermediate $\mathrm{K}$ content, and about $0.9 \%$ for alkali-rich basalt (Moore, 1970). Lowest $\mathrm{H}_{2} \mathrm{O}^{+}$values, in the range $0.8 \%-0.9 \%$, are for probable pillow glasses at Sites 266 and 267. These glasses, also, are among the leastoxidized samples, though all but glass (266-23-1-4) show significant oxidation. Assuming that all samples at each site are derived from a single, original, homogeneous magma at the site and that effects of crystal accumulation are negligible-both assumptions being in accord with available evidence-and that sea-floor weathering can be inferred from oxidation (Cann, 1971), weathering has produced appreciable changes in amounts of some major elements such as $\mathrm{K}$ but not in others such as $\mathrm{Na}$ 
TABLE 15

Minor-Element Content of Basalt From Site $274^{\mathrm{a}}$

\begin{tabular}{|c|c|c|c|c|c|c|}
\hline $\begin{array}{l}\text { Sample } \\
\text { Plot Symbol }\end{array}$ & $\underset{P}{44-1-2}$ & $\begin{array}{c}44-2-15 \\
Q\end{array}$ & $\begin{array}{c}44-2-18 \\
R\end{array}$ & $\begin{array}{c}44, \mathrm{CC} \\
\mathrm{S}\end{array}$ & $\begin{array}{c}45-2-35 \\
T\end{array}$ & $\begin{array}{c}45-2-39 \\
U\end{array}$ \\
\hline $\begin{array}{l}\mathrm{Ag} \\
\mathrm{Au}(\mathrm{ppb}) \\
\mathrm{B} \\
\mathrm{Ba} \\
\mathrm{Ce}\end{array}$ & $\begin{array}{l}0.03 \\
0.49 \\
\mathrm{~N} \\
13 \\
12\end{array}$ & $\begin{array}{l}0.07 \\
0.06 \\
N \\
40 \\
11\end{array}$ & $\begin{array}{c}0.03 \\
0.39 \\
\mathrm{~N} \\
32 \\
8\end{array}$ & $\begin{array}{l}0.03 \\
0.74 \\
N \\
26 \\
12\end{array}$ & $\begin{array}{c}0.01 \\
0.84 \\
70 \\
100 \\
38.1\end{array}$ & $\begin{array}{c}0.02 \\
0.3 \\
40 \\
180 \\
34.8\end{array}$ \\
\hline $\begin{array}{l}\mathrm{Co} \\
\mathrm{Cr} \\
\mathrm{Cr} * \\
\mathrm{Cu} \\
\mathrm{Eu}\end{array}$ & $\begin{array}{c}50 \\
280 \\
127 \\
90 \\
1.0\end{array}$ & $\begin{array}{c}40 \\
220 \\
134 \\
90 \\
1.5\end{array}$ & $\begin{array}{c}40 \\
260 \\
127 \\
75 \\
1.0\end{array}$ & $\begin{array}{c}40 \\
220 \\
123 \\
85 \\
1.2\end{array}$ & $\begin{array}{c}19 \\
260 \\
150 \\
70 \\
1.4\end{array}$ & $\begin{array}{r}26 \\
260 \\
135 \\
110 \\
1.2\end{array}$ \\
\hline $\begin{array}{l}\mathrm{Ga} \\
\mathrm{Hf} \\
\mathrm{La} \\
\mathrm{Mn} \\
\mathrm{Nd}\end{array}$ & $\begin{array}{c}22 \\
2.7 \\
\mathrm{~N} \\
1200 \\
14\end{array}$ & $\begin{array}{c}15 \\
2.8 \\
\mathrm{~N} \\
900 \\
16\end{array}$ & $\begin{array}{c}15 \\
2.6 \\
N \\
700 \\
7\end{array}$ & $\begin{array}{c}15 \\
2.5 \\
\mathrm{~N} \\
460 \\
10\end{array}$ & $\begin{array}{c}15 \\
3.4 \\
\mathrm{~N} \\
650 \\
13\end{array}$ & $\begin{array}{c}13 \\
3.5 \\
\mathrm{~N} \\
1100 \\
16\end{array}$ \\
\hline $\begin{array}{l}\mathrm{Ni} \\
\mathrm{Pb} \\
\mathrm{S} \\
\mathrm{Sb} \\
\mathrm{Sc}\end{array}$ & $\begin{array}{r}100 \\
\mathrm{~N} \\
1300 \\
\mathrm{~N} \\
48\end{array}$ & $\begin{array}{r}80 \\
\mathrm{~N} \\
1400 \\
\mathrm{~N} \\
50\end{array}$ & $\begin{array}{r}90 \\
\mathrm{~N} \\
1200 \\
\mathrm{~N} \\
50\end{array}$ & $\begin{array}{c}100 \\
\mathrm{~N} \\
1200 \\
0.5 \\
40\end{array}$ & $\begin{array}{l}50 \\
\mathrm{~N} \\
0 \\
0.5 \\
30\end{array}$ & $\begin{array}{r}50 \\
\mathrm{~N} \\
400 \\
\mathrm{~N} \\
40\end{array}$ \\
\hline $\begin{array}{l}\mathrm{Sr} \\
\mathrm{Ta} \\
\mathrm{Th} \\
\mathrm{Th} \\
\mathrm{U}\end{array}$ & $\begin{array}{c}130 \\
0.3 \\
\mathrm{~N} \\
<1 \\
0.19\end{array}$ & $\begin{array}{l}140 \\
\quad 0.4 \\
N \\
N \\
0.74\end{array}$ & $\begin{array}{l}130 \\
\quad 0.3 \\
\mathrm{~N} \\
\mathrm{~N} \\
0.28\end{array}$ & $\begin{array}{l}140 \\
0.3 \\
\mathrm{~N} \\
\mathrm{~N} \\
0.16\end{array}$ & $\begin{array}{l}250 \\
1.4 \\
\mathrm{~N} \\
\mathrm{~N} \\
0.51\end{array}$ & $\begin{array}{l}220 \\
1.4 \\
2.7 \\
1 \\
0.39\end{array}$ \\
\hline $\begin{array}{l}\mathrm{V} \\
\mathrm{Y} \\
\mathrm{Yb} \\
\mathrm{Yb}{ }^{*} \\
\mathrm{Zr}\end{array}$ & $\begin{array}{c}340 \\
50 \\
5 \\
4.0 \\
100\end{array}$ & $\begin{array}{c}300 \\
50 \\
3 \\
3.9 \\
100\end{array}$ & $\begin{array}{c}300 \\
60 \\
3 \\
3.6 \\
100\end{array}$ & $\begin{array}{c}340 \\
50 \\
3 \\
3.4 \\
100\end{array}$ & $\begin{array}{c}250 \\
40 \\
3 \\
3.8 \\
140\end{array}$ & $\begin{array}{c}220 \\
50 \\
3 \\
3.5 \\
140\end{array}$ \\
\hline
\end{tabular}

${ }^{\mathrm{a}} \mathrm{See}$ text for analysts and methods; all values in ppm except for $\mathrm{Au}$ in ppb; N, element sought but below detectability limits.

(Figure 2). Not all of these changes are in accord with results reported by Melson (1973), as described earlier in the discussion of chemistry of Site 267 basalt. Except for $\mathrm{Al}_{2} \mathrm{O}_{3}$, major-element alterations of Leg 28 basalt are generally similar to those reported by Hart et al. (1974).

Effects at some sites of inferred alteration on minorelement abundances are discussed earlier. Alteration has apparently contributed to diversity in some minor element abundances but not in others. In contrast to Aumento's (1971) finding of U enrichment by alteration of Mid-Atlantic Ridge tholeiite, analyses of Leg 28 basalts support Thompson's (1973) conclusion that U concentrations appear to be independent of alteration (Figure 4 . S concentrations, averaging only $140 \mathrm{ppm}$, are much lower in southeast Indian Ocean basalts than the $800 \pm 150 \mathrm{ppm}$ estimated by Moore and Fabbi (1971) as the juvenile $\mathrm{S}$ content of mantle-melt basalt, and than the average $917 \mathrm{ppm}$ of Site 274 basalt. Variation in S abundance does not appear to relate to oxidation. Although at Site $265 \mathrm{Ni} / \mathrm{Co}$ ratios appear to decrease with alteration, at all other sites there appears to be little relationship between $\mathrm{Ni} / \mathrm{Co}$ and alteration (Figure 5). Abundances of $\mathrm{Ag}$ and $\mathrm{Au}$ are not well known in DSDP basalts. Leg $28 \mathrm{Au}$ values are slightly lower than in low$\mathrm{K}$ tholeiite from the East Pacific Rise (Gottfried et al., 1972), and $\mathrm{Ag}$ contents approximate those of mafic rocks reported by Greenland and Fones (1971). The rare-earth element, Eu, is comparatively low in abundance at Site 267 , but values generally approximate those of Mid-Atlantic Ridge tholeiite (Frey et al., 1968) and show little effect of alteration.

Certain minor elements such as $\mathrm{TiO}_{2}, \mathrm{P}_{2} \mathrm{O}_{5}, \mathrm{Zr}$, and $\mathrm{Sr}$ appear to resist alteration processes better than others, according to Bass et al. (1973), and their distributions in variation diagrams fall into three rather well-defined groupings of alkali, ocean-island, and ocean-ridge basalts. At Leg 28 Sites 265, 266, and 267 where all samples appear to be from a single flow unit, concentrations of these elements show no apparent systematic variation with oxidation index. Plots of $\mathrm{P}_{2} \mathrm{O}_{5}$ and $\mathrm{TiO}_{2}$ (Figure 11) for all Leg 28 basalts except the two most altered Site 274 rocks lie within or very near the field for ocean-ridge basalt of Bass et al. (1973). Site 266 rocks show the most alkalic tendency in this plot. In terms of $\mathrm{Zr}$ and $\mathrm{Sr}$ (Figure 12) most analyses also lie within the ocean-ridge field except for those of Site 265 which contain unusually high $\mathrm{Sr}$ and plot within the ocean-island basalt field. High $\mathrm{Sr}$ abundance does not appear to relate to possible calcium carbonate introduction as inferred from $\mathrm{CO}_{2}$ and $\mathrm{CaO}$ contents.

The subalkaline character of most Leg 28 basalts is shown in the alkali-silica diagram of Figure 13. If nor- 
TABLE 16

Selected Characteristics of Site 274 Basalts

\begin{tabular}{|c|c|c|c|c|c|c|}
\hline $\begin{array}{l}\text { Sample } \\
\text { Plot Symbol }\end{array}$ & $\begin{array}{c}44-1-2 \\
P\end{array}$ & $\begin{array}{c}44-2-15 \\
Q\end{array}$ & $\begin{array}{c}44-2-18 \\
\mathrm{R}\end{array}$ & $\begin{array}{c}44, \mathrm{CC} \\
\mathrm{S}\end{array}$ & $\begin{array}{c}45-2-35 \\
T\end{array}$ & $\begin{array}{c}45-2-39 \\
U\end{array}$ \\
\hline \multicolumn{7}{|l|}{ Mode } \\
\hline Plagioclase & 32.3 & 31.7 & 47.1 & 47.6 & n.d. & n.d. \\
\hline Clinopyroxene & - & - & 18.1 & 16.8 & & \\
\hline Opaque oxide & 0.4 & 0.5 & 4.9 & 4.4 & & \\
\hline \multicolumn{4}{|l|}{$\begin{array}{l}\text { Fine matrix, } \\
\text { incl. pyroxene }\end{array}$} & 31.2 & & \\
\hline Rock color & $\begin{array}{c}\text { Med 1t } \\
\text { gray, N6 }\end{array}$ & $\begin{array}{c}\text { Dk greenish } \\
\text { gray } 5 \mathrm{GY} 4 / 1\end{array}$ & \multicolumn{4}{|c|}{ All same as 44-2-15 } \\
\hline Density $(\mathrm{g} / \mathrm{cc})$ & 2.78 & 2.71 & 2.65 & 2.69 & 2.49 & 2.59 \\
\hline \multicolumn{7}{|c|}{ Plagioclase compositions } \\
\hline Normative & 59.0 & 59.2 & 55.8 & 58.1 & 50.6 & 41.4 \\
\hline \multicolumn{7}{|l|}{ Ratios } \\
\hline $\mathrm{Ba} / \mathrm{Sr}$ & 0.10 & 0.29 & 0.25 & 0.19 & 0.40 & 0.82 \\
\hline $\mathrm{Ni} / \mathrm{Co}$ & 5.0 & 2.0 & 2.3 & 2.5 & 2.6 & 1.9 \\
\hline $\mathrm{Na} / \mathrm{K}$ & 8.9 & 11.5 & 7.3 & 6.2 & 1.7 & 1.7 \\
\hline $\mathrm{K} / \mathrm{Zr}$ & 22 & 17 & 31 & 35 & 89 & 89 \\
\hline $\mathrm{K} / \mathrm{Ba}$ & 172 & 44 & 96 & 134 & 125 & 69 \\
\hline $\mathrm{K} / \mathrm{Cr} *$ & 18 & 13 & 24 & 28 & 83 & 92 \\
\hline Oxidation index ${ }^{\mathrm{a}}$ & 32.6 & 41.0 & 48.7 & 52.1 & 72.3 & 67.0 \\
\hline $\mathrm{FeO}^{*} / \mathrm{MgO}$ & 2.1 & 1.4 & 1.3 & 1.2 & 1.6 & 1.4 \\
\hline Mafic index ${ }^{a}$ & 68.7 & 59.5 & 57.1 & 56.6 & 62.7 & 59.5 \\
\hline D.I. ${ }^{\mathrm{a}}$ & 32.5 & 31.7 & 33.4 & 33.0 & 40.2 & 42.2 \\
\hline
\end{tabular}

${ }^{\text {a }}$ See text.

malized free of volatiles, all would lie within the tholeiite field of Irvine and Baragar (1971) and most would also lie within the more restricted tholeiite field of Macdonald (1968). Site 267 basalts closely compare in this diagram with average oceanic tholeiite of Engel et al. (1965) and with average DSDP Leg 26 southern Indian Ocean basalt reported by Kempe (1973). Quartz normativeness is the main chemical characteristic of southern Indian Ocean basalt (Kempe, 1973) and is a feature shared by Site 267 as well as Site 274 rocks (Table 18). As a group, Site 265 basalts are the most alkalic (Figure 13).

Plots of relative molecular proportions of $\mathrm{Q}, \mathrm{Di}, \mathrm{Ol}$, and $\mathrm{Ne}$ (Tables 2, 6, 10,14, 18), following the method of Coombs (1963), also indicate that Site 265 basalts are the most alkalic of Leg 28 rocks (Figure 7). In Figure 7, points by letter symbols show plots of values based on analyzed oxide amounts. Lines connect these points to those where the analyses plot after reducing excess $\mathrm{Fe}_{2} \mathrm{O}_{3}$ to $\mathrm{FeO}$. The lines indicate probable range of original composition; oxidation produces rocks with more tholeiitic characteristics than originally present. Relative apparent "alkalinity" is quantified using Coombs' (1963) "indicator ratios," which are values on the Di-Q join projected from the line connecting the $\mathrm{Ol}$ apex and the plotted sample. According to this diagram, average original Site 266 rocks are the least alkaline of southeast Indian Ocean basalts and both they and Site 267 basalts have typically tholeiitic indicator ratios of 0.50 or greater. Average Site 265 rocks are distinctly more alkaline, and Site 274 rocks are much more tholeiitic. Although present Site 265 rocks, having an average indicator ratio of 0.45 , are less alkaline than average oceanic tholeiite, with a ratio of 0.40 , reducing excess $\mathrm{Fe}_{2} \mathrm{O}_{3}$ shows them to be considerably more alkaline, having a mildly alkaline indicator ratio of about 0.25 . The occurrence of groundmass and microphenocrystic olivine, as in Site 265 rocks, is characteristic for basalts having ratios of 0.00-0.38 (Coombs, 1963). Although basalts with ratios exceeding 0.50 , such as those of Sites 266, 267, and 274, typically contain hypersthene phenocrysts (Coombs, 1963), none was observed in the Leg 28 rocks.

Parent-magma compositions for southeast Indian Ocean basalts probably lie near the cotectic curve in the normative olivine-plagioclase-pyroxene diagram (Figure 14) of Shido et al. (1971). Oxidation-adjusted norm values are used in this plot. Rocks plotted are those that appear to be the least altered at each site. The Site 265 sample is holocrystalline, carrying rare microphenocrysts of both olivine and plagioclase, but is classed as OL tholeiite (Shido et al., 1971) because glassy rocks in the suite indicate that olivine was the first to crystallize. The Site 266 sample is aphyric glass and so cannot be petrographically classed as either OL or PL tholeiite. Sites 267 and 274 are PL tholeiites containing phenocrystic plagioclase, abundantly at Site 274 . Of the plots in Figure 14, that for Site 274 has the least probability of representing original magma composition as it is a porphyritic holocrystalline rock with no associated glassy 
TABLE 17

Comparison of Average Leg 28 Basalts: Major Oxides

\begin{tabular}{|c|c|c|c|c|c|c|c|c|}
\hline & $\begin{array}{c}\text { Site } \\
265^{\mathrm{a}}\end{array}$ & $\begin{array}{l}\text { Site } \\
266^{b}\end{array}$ & $\begin{array}{l}\text { Site } \\
267^{c}\end{array}$ & $\begin{array}{l}\text { Average } \\
\text { SE Indiagn } \\
\text { Ocean }\end{array}$ & $\begin{array}{l}\text { Site } \\
274 \mathrm{e}\end{array}$ & $\begin{array}{l}\text { Average } \\
\text { SE Indian } \\
\text { Oceanf }\end{array}$ & $\begin{array}{c}\text { Oceanic } \\
\text { Tholeiiteg }\end{array}$ & $\begin{array}{l}\text { Alkali } \\
\text { Basalt }\end{array}$ \\
\hline $\mathrm{SiO}_{2}$ & 47.7 & 48.8 & 50.5 & 48.6 & 48.4 & 49.16 & 49.34 & 47.41 \\
\hline $\mathrm{A}_{2}{ }_{2} \mathrm{O}_{3}$ & 16.7 & 14.8 & 15.4 & 15.8 & 16.6 & 14.86 & 17.04 & 18.02 \\
\hline $\mathrm{Fe}_{2} \mathrm{O}_{3}$ & 5.1 & 4.6 & 3.8 & 4.7 & 4.5 & 2.61 & 1.99 & 4.17 \\
\hline $\mathrm{FeO}$ & 3.7 & 6.7 & 5.4 & 5.0 & 4.0 & 7.80 & 6.82 & 5.80 \\
\hline $\mathrm{MgO}$ & 7.5 & 6.3 & 7.3 & 7.1 & 5.5 & 7.30 & 7.19 & 4.79 \\
\hline $\mathrm{CaO}$ & 10.4 & 10.2 & 11.7 & 10.6 & 9.0 & 11.10 & 11.72 & 8.65 \\
\hline $\mathrm{Na}_{2} \mathrm{O}$ & 3.0 & 2.7 & 2.3 & 2.8 & 2.9 & 2.40 & 2.73 & 3.99 \\
\hline $\mathrm{K}_{2} \mathrm{O}$ & 0.76 & 0.58 & 0.43 & 0.63 & 0.71 & 0.17 & 0.16 & 1.66 \\
\hline $\mathrm{H}_{2} \mathrm{O}+$ & 1.4 & 1.2 & 0.87 & 1.2 & 1.8 & 0.91 & 0.69 & 0.79 \\
\hline $\mathrm{H}_{2} \mathrm{O}-$ & 1.3 & 1.1 & 0.60 & 1.1 & 3.4 & 1.23 & 0.58 & 0.61 \\
\hline $\mathrm{TiO}_{2}$ & 1.6 & 2.0 & 1.3 & 1.7 & 1.7 & 1.63 & 1.49 & 2.87 \\
\hline $\mathrm{P}_{2} \mathrm{O}_{5}$ & 0.28 & 0.28 & 0.16 & 0.26 & 0.25 & 0.18 & 0.16 & 0.92 \\
\hline $\mathrm{MnO}$ & 0.16 & 0.22 & 0.20 & 0.19 & 0.15 & 0.19 & 0.17 & 0.16 \\
\hline $\mathrm{CO}_{2}$ & 0.41 & 0.32 & 0.01 & 0.30 & 1.3 & 0.51 & - & - \\
\hline \multicolumn{9}{|l|}{ Oxidation } \\
\hline index & 58.0 & 40.7 & 41.3 & 48.5 & 52.9 & 25.1 & 22.6 & 41.8 \\
\hline $\mathrm{FeO} * / \mathrm{MgO}$ & 1.1 & 1.7 & 1.2 & 1.3 & 1.5 & 1.4 & 1.2 & 2.0 \\
\hline Mafic index & 54.0 & 64.2 & 55.8 & 57.7 & 60.7 & 58.8 & 55.1 & 67.5 \\
\hline D.I. & 30.3 & 29.8 & 25.6 & 29.0 & 36.0 & 23.5 & 24.2 & 42.8 \\
\hline
\end{tabular}

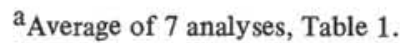

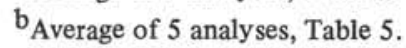

c Average of 3 analyses, Table 9.

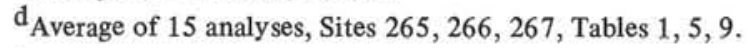

eAverage of 6 analyses, Table 13.

${ }^{f}$ Average basalt, Southern Indian Ocean, Leg 26 (Kempe, 1973).

grom Engel et al. (1965).
}

rocks for comparison. Figure 14 shows the considerable probable diversity in original magma compositions of the southeast Indian Ocean basalts.

Minor-element distributions generally do not follow systematic trends from site to site (Figures 11 and 12). In contrast, in suites of related rocks, $\mathrm{Ni} / \mathrm{Co}$ ratios plotted against mafic index follow rather well-defined systematic trends though differing for different suites (Fleischer, 1968). Ni/Co ratios plotted against mafic index of Leg 28 basalts (Figure 15) show no systematic variation from site to site comparable to that of other basaltic suites shown, which suggests that compositional variation is not the result of near-surface fractionation of the same source magma.

\section{CONCLUSIONS}

Basalts of the southeast Indian Ocean have generally typical ocean-floor tholeiite compositions that differ in major oxides from typical ridge-axis basalt mainly in their relatively high $\mathrm{K}_{2} \mathrm{O}$ content. Ridge-axis basalts are almost universally olivine tholeiites that except for lower $\mathrm{K}_{2} \mathrm{O}$ have compositions approximating those at Site 265 (Kay et al., 1970), the nearest site to the axis of the
Southeast Indian Ridge. Site 267, the farthest, has $\mathrm{K}_{2} \mathrm{O}$ content closer to MORB values. In terms of major elements and some minor elements, the Site 265 basalts seem to have more alkaline characteristics than those farther down the ridge flank at Site 266 and at Site 267 in the South Indian Basin (Figures 7, 12,13). Rocks with the characteristic low-K MORB compositions may be restricted to a narrow axial zone of the ridge which has not been sampled; and chemical differences between Site 265 basalts and typical MORB and between Sites 265, 266 , and 267 may be interpreted in terms of the basaltic magma intrusion model of Kay et al. (1970). The chemical characteristics suggest that Leg 28 basalts may be ridge-flank and ocean-basin eruptives rather than ridge-axis basalts that moved laterally by a sea-floorspreading mechanism. However, as basalt penetration was only superficial at all drill sites, more typical ridgeaxis basalt may exist at greater depth.

The tectonic setting of Site 274 near Balleny Basin is uncertain. Site 274 basalts show major chemical differences (Figures 2, 7, 14) from southeast Indian Ocean basalts but they also show some important similarities (Figures 2, 11-13). Most southeast Indian 
TABLE 18

Comparison of Average Leg 28 Basalts: Norms

\begin{tabular}{|c|c|c|c|c|c|c|c|c|}
\hline & Site 265 & Site 266 & Site 267 & $\begin{array}{l}\text { Average } \\
\text { SE Indian } \\
\text { Ocean }\end{array}$ & Site 274 & $\begin{array}{l}\text { Average } \\
\text { S Indian } \\
\text { Ocean }\end{array}$ & $\begin{array}{l}\text { Oceanic } \\
\text { Tholeiitic }\end{array}$ & $\begin{array}{l}\text { Alkali } \\
\text { Basalt }\end{array}$ \\
\hline Q & - & $3.1(-)$ & $3.5(0.8)$ & $1.3(-)$ & $6.3(2.8)$ & $2.0(0.7)$ & - & - \\
\hline Or & 4.6 & 3.5 & 2.6 & 3.8 & 4.3 & 1.0 & 1.0 & 9.9 \\
\hline $\mathrm{Ab}$ & 25.7 & 23.1 & 19.6 & 24.0 & 25.3 & 20.6 & 23.2 & 31.5 \\
\hline An & 30.2 & 26.9 & 30.6 & 29.0 & 31.3 & 29.6 & 33.9 & 26.6 \\
\hline $\mathrm{Ne}$ & - & - & - & - & - & - & - & $1.2(3.2)$ \\
\hline (Wo & $7.3(7.5)$ & $8.5(8.7)$ & 11.1(11.3) & $8.6(8.7)$ & $2.0(2.0)$ & $9.0(9.1)$ & $9.8(9.9)$ & $4.4(4.5)$ \\
\hline Di $\{$ En & $6.3(4.7)$ & $5.8(4.6)$ & $8.0(6.7)$ & $6.6(5.1)$ & $1.6(1.2)$ & $5.5(5.2)$ & $6.2(6.0)$ & $3.3(2.4)$ \\
\hline CFs & $0.1(2.3)$ & $2.1(3.9)$ & $2.2(4.0)$ & $1.1(3.1)$ & $0.1(0.7)$ & $3.0(3.6)$ & $3.0(3.3)$ & $0.8(1.9)$ \\
\hline $\mathrm{Hy}\left\{{ }^{\text {En }}\right.$ & $10.7(3.0)$ & $10.1(10.7)$ & $10.3(11.8)$ & $11.3(7.4)$ & $12.5(13.3)$ & $12.9(13.4)$ & $8.2(7.3)$ & - \\
\hline Fs & $0.1(1.4)$ & $3.6(9.1)$ & $2.8(7.0)$ & $1.8(4.6)$ & $1.0(8.3)$ & $7.0(9.3)$ & $4.0(4.1)$ & - \\
\hline Ol $\left\{\begin{array}{l}\text { Fo } \\
\end{array}\right.$ & $1.4(8.1)$ & $-(0.6)$ & - & $-(3.9)$ & - & - & $2.5(3.4)$ & $6.1(6.8)$ \\
\hline$l_{\mathrm{Fa}}$ & $0.0(4.4)$ & $-(0.6)$ & - & $-(2.6)$ & - & - & $1.4(2.1)$ & $1.6(5.9)$ \\
\hline Mt & $7.5(2.2)$ & $6.8(2.2)$ & $5.5(2.2)$ & $6.9(2.2)$ & $6.7(2.3)$ & $3.8(2.2)$ & $2.9(2.2)$ & $6.1(2.2)$ \\
\hline II & 3.1 & 3.8 & 2.5 & 3.3 & 3.3 & 3.1 & 2.8 & 5.5 \\
\hline Ap & 0.7 & 0.7 & 0.4 & 0.6 & 0.6 & 0.4 & 0.4 & 2.2 \\
\hline $\mathrm{Cc}$ & 0.9 & 0.7 & 0.0 & 0.7 & 3.1 & 1.2 & - & - \\
\hline $\begin{array}{l}\text { Plagio- } \\
\text { clase \%An }\end{array}$ & 54.0 & 53.7 & 61.0 & 54.8 & 55.2 & 59.0 & 59.4 & 45.8 \\
\hline \multicolumn{9}{|c|}{ Molecular proportions Ne:Di:Hy:OI:Q (recalculated to $100 \%$ ) } \\
\hline $\mathrm{Ne}$ & - & - & - & - & - & - & - & $5.1(8.9)$ \\
\hline $\mathrm{Di}$ & $34.9(34.9)$ & $29.0(29.0)$ & $34.5(34.5)$ & $33.4(33.4)$ & $6.7(6.7)$ & $26.7(26.7)$ & $38.1(38.1)$ & $40.5(30.3)$ \\
\hline Hy & $59.6(22.0)$ & $50.4(68.3)$ & $44.8(60.5)$ & $57.1(48.5)$ & $52.1(75.0)$ & $62.1(69.4)$ & $50.8(46.6)$ & - \\
\hline $\mathrm{Ol}$ & $5.5(43.1)$ & $-(2.7)$ & - & $-(18.1)$ & - & - & $11.1(15.3)$ & $54.4(60.8)$ \\
\hline Q & - & $20.6(0)$ & $20.7(5.0)$ & $9.5(0)$ & $41.2(18.3)$ & $11.2(3.9)$ & - & - \\
\hline
\end{tabular}

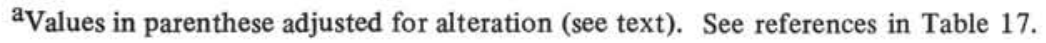

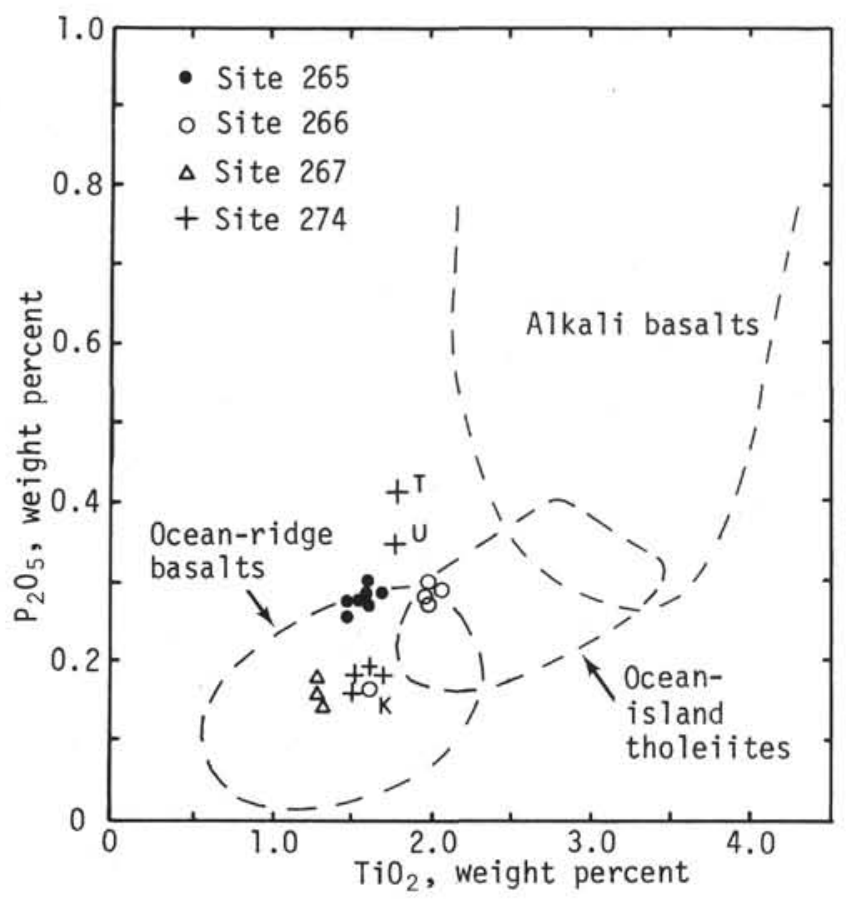

Figure 11. Plots of $\mathrm{TiO}_{2}$ and $\mathrm{P}_{2} \mathrm{O}_{5}$ values for Leg 28 basalts. Fields for basalt types from Bass et al. (1973).
Ocean basalts and all Site 274 basalts lie within the ocean-floor basalt field in a $\mathrm{Ti}-\mathrm{Zr}$ diagram, not shown, of Pearce and Cann (1973). In a Ti-Zr-Y ternary plot, also not shown, Site 274 basalts form a well-defined grouping in the "within-plate" basalt field of Pearce and Cann (1973), distinctly apart from southeast Indian

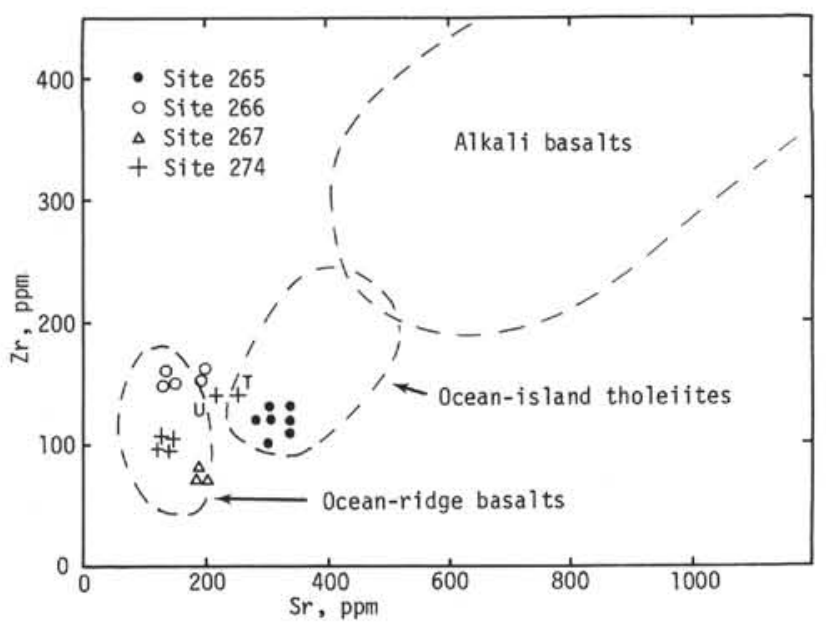

Figure 12. Plots of $\mathrm{Sr}$ and $\mathrm{Zr}$ values for Leg 28 basalts. Fields for basalt types from Bass et al. (1973). 
TABLE 19

Comparison of Average Leg 28 Basalts: Minor Elements ${ }^{\mathrm{a}}$

\begin{tabular}{|c|c|c|c|c|c|c|c|}
\hline & $\begin{array}{l}\text { Site } \\
265^{\mathrm{a}}\end{array}$ & $\begin{array}{l}\text { Site } \\
266^{b}\end{array}$ & $\begin{array}{l}\text { Site } \\
267^{c}\end{array}$ & $\begin{array}{l}\text { Average } \\
\text { SE Indiąn } \\
\text { Ocean }\end{array}$ & $\begin{array}{l}\text { Site } \\
274^{\mathrm{e}}\end{array}$ & $\begin{array}{l}\text { Oceanic } \\
\text { Tholeiiteg }\end{array}$ & $\begin{array}{l}\text { Alkali } \\
\text { Basaltg }\end{array}$ \\
\hline $\mathrm{Ag} \mathrm{ppm}$ & 0.07 & 0.09 & 0.06 & 0.07 & 0.03 & & \\
\hline $\mathrm{Au} \mathrm{ppb}$ & 0.4 & 0.4 & 0.2 & 0.4 & 0.5 & & \\
\hline Ba ppm & 84 & 40 & 10 & 55 & 63.5 & 14 & 498 \\
\hline $\mathrm{Ce} \mathrm{ppm}$ & 20.5 & 19.1 & 9 & 17.7 & 19 & & \\
\hline Co ppm & 38 & 39 & 41 & 39 & 36 & 32 & 25 \\
\hline $\mathrm{Cr} * \mathrm{ppm}$ & 312 & 151 & 175 & 231 & 133 & 297 & 67 \\
\hline $\mathrm{Cu} \mathrm{ppm}$ & 62 & 64 & 60 & 62 & 87 & 77 & 36 \\
\hline Eu ppm & 1.3 & 1.5 & 0.9 & 1.3 & 1.2 & & \\
\hline Ga ppm & 16 & 18 & 17 & 17 & 16 & 17 & 22 \\
\hline Hf ppm & 2.7 & 3.6 & 2.1 & 2.9 & 2.9 & & \\
\hline Mn ppm & 850 & 1180 & 1050 & 1000 & 835 & & \\
\hline $\mathrm{Nd} \mathrm{ppm}$ & 14 & 16 & 10 & 14 & 13 & & \\
\hline $\mathrm{Ni}$ ppm & 210 & 122 & 83 & 155 & 78 & 97 & 51 \\
\hline S ppm & 100 & 280 & 0 & 140 & 917 & & \\
\hline $\mathrm{Sb} \mathrm{ppm}$ & 0 & 0 & 0.3 & 0.1 & 0 & & \\
\hline Sc ppm & 33 & 41 & 45 & 38 & 43 & 61 & 26 \\
\hline Sr ppm & 314 & 156 & 193 & 237 & 168 & 130 & 815 \\
\hline Ta ppm & 0.7 & 0.4 & 0.2 & 0.5 & 0.7 & & \\
\hline U ppm & 0.37 & 0.28 & 0.29 & 0.33 & 0.38 & & \\
\hline V ppm & 180 & 316 & 287 & 247 & 292 & .292 & 252 \\
\hline Y ppm & 44 & 56 & 40 & 47 & 50 & & \\
\hline $\mathrm{Yb}^{*} \mathrm{ppm}$ & 2.8 & 5.4 & 3.3 & 3.8 & 3.7 & 5 & 4 \\
\hline $\mathrm{Zr} \mathrm{ppm}$ & 119 & 154 & 77 & 122 & 113 & 95 & 333 \\
\hline $\mathrm{Ba} / \mathrm{Sr}$ & 0.27 & 0.26 & 0.05 & 0.23 & 0.38 & 0.11 & 0.61 \\
\hline $\mathrm{Ni} / \mathrm{Co}$ & 5.5 & 3.1 & 2.0 & 4.0 & 2.2 & 3.0 & 2.0 \\
\hline $\mathrm{Na} / \mathrm{K}$ & 3.5 & 4.2 & 4.8 & 4.0 & 3.6 & 15.6 & 2.1 \\
\hline $\mathrm{K} / \mathrm{Zr}$ & 53 & 31 & 47 & 43 & 52 & 14 & 41 \\
\hline $\mathrm{K} / \mathrm{Ba}$ & 75 & 120 & 360 & 95 & 93 & 93 & 28 \\
\hline $\mathrm{K} / \mathrm{Cr}$ & 20 & 32 & 21 & 23 & 44 & 4 & 206 \\
\hline
\end{tabular}

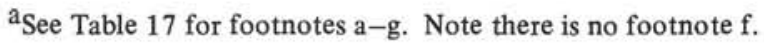

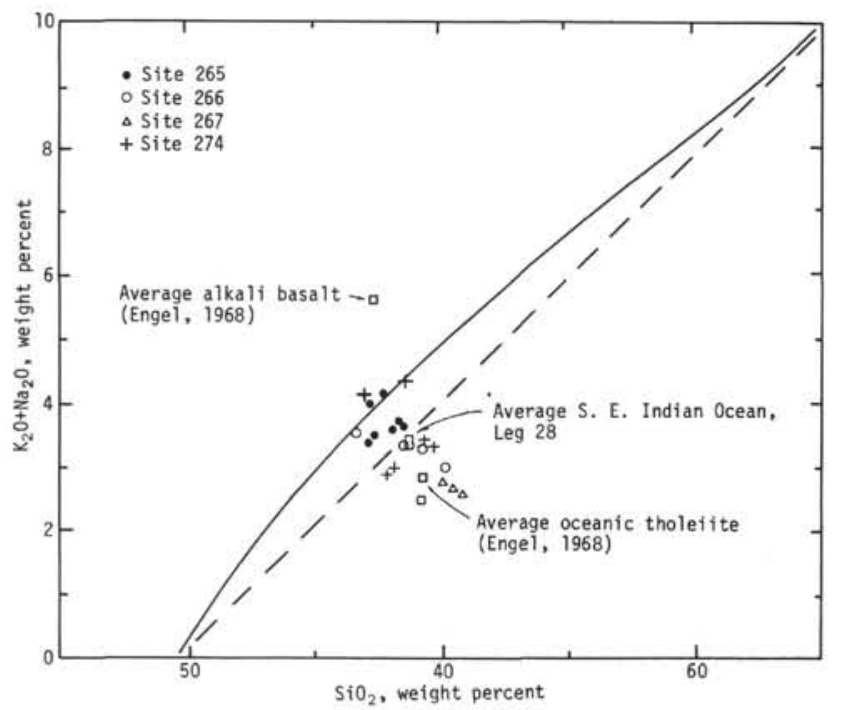

Figure 13. Comparison of Leg 28 basalts in alkali-silica diagram showing divisions between alkalic and tholeiitic fields of Irvine and Baragar (1971), solid line, and of Macdonald (1968), dashed line.

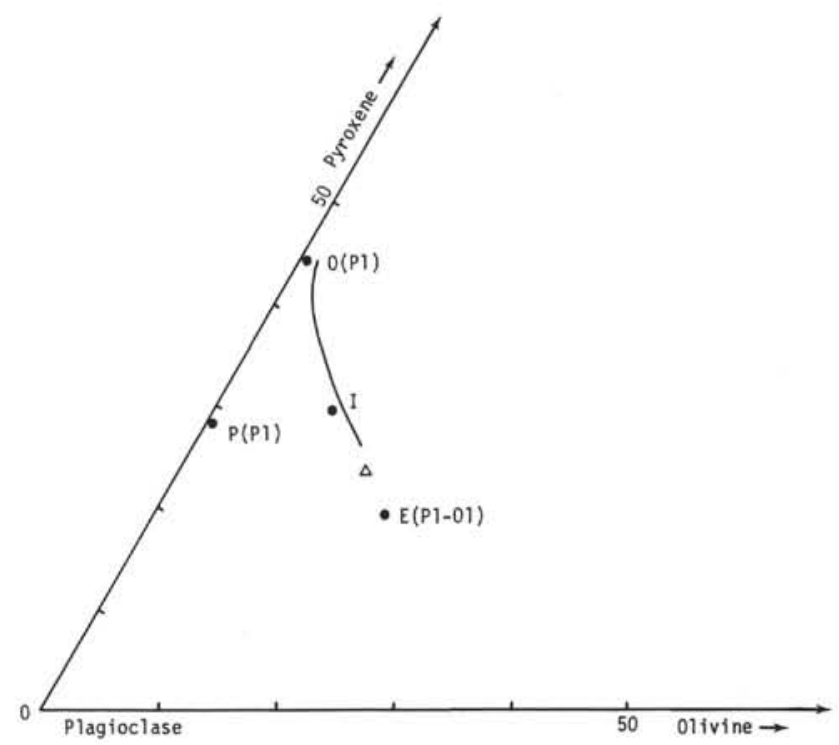

Figure 14. Normative olivine-plagioclase-pyroxene diagram showing cotectic curve of Shido et al. (1971) and probable magma compositions of Leg 28 basalt. $O L$ and $P L$, in parentheses, identifies probable first phenocrystic phase to crystallize. 


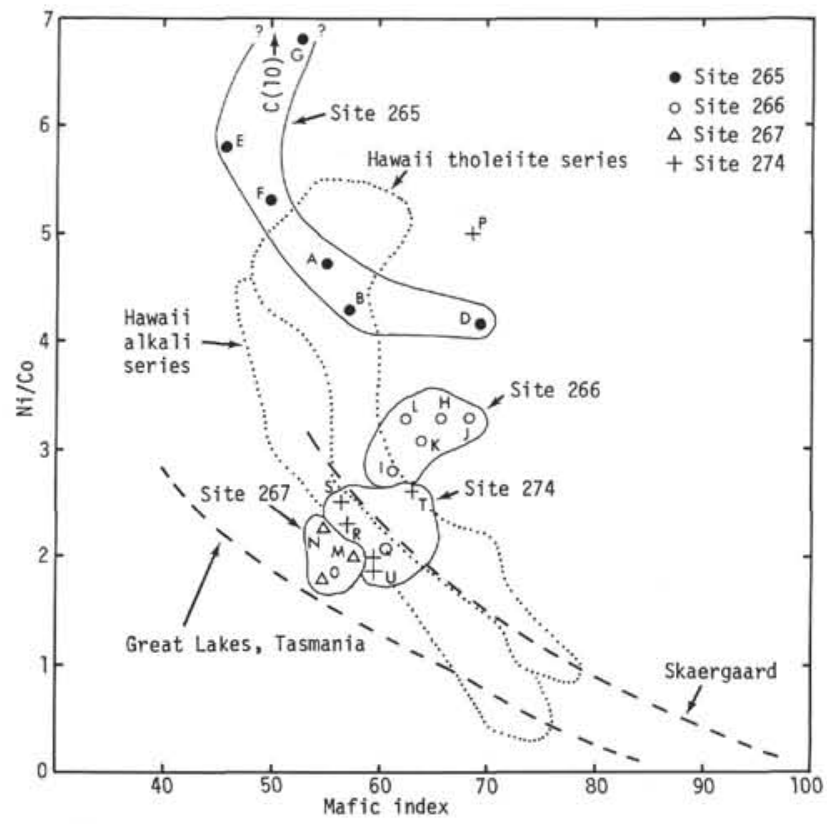

Figure 15. Plots of Ni/Co ratio versus mafic index for Leg 28 basalts compared with trends of basaltic suites shown by Fleischer (1968).

Ocean basalts. The "within-plate" field includes oceanisland and continental basalts, according to Pearce and Cann (1973). The minor-element data, however, conflicts with other criteria (Figures 11 and 12) which are suggestive of ocean-ridge characteristics. The great disparity of compositional plots in Figure 7, and the comparatively high degree of silica saturation (Table 18) are suggestive of a nonridge origin of Site 274 basalt. In absence of more definitive criteria, these basalts are believed to represent "oceanic-island," or seamount, rocks possibly related to an early stage of development of the nearby Balleny Islands volcanic complex whose latest eruptions were of alkaline olivine basalt (Mawson, 1950).

\section{ACKNOWLEDGMENTS}

I am indebted to all fellow shipboard members of Leg 28 of D/V Glomar Challenger as well as to analysts of the U.S. Geological Survey for shore-based studies. In particular, I am indebted to P.J. Barrett and E.M. Kemp, who contributed to shipboard study of the basalt core, and to R.G. Coleman and N.S. MacLeod, who reviewed the manuscript.

\section{REFERENCES}

Aumento, F., 1971. Uranium content of mid-ocean basalts: Earth Plan. Sci. Lett., v. 11, p. 90.

Bass, M.N., Moberly, R.M., Rhodes, J.M., Shih, Chi-yu, and Church, S.E., 1973. Volcanic rocks cored in the central Pacific, Leg 17, Deep Sea Drilling Project. In Winterer, E.L., Ewing, J.I., et al., Initial Reports of the Deep Sea Drilling Project, Volume 17: Washington (U.S. Government Printing Office), p. 429.

Cann, J.R., 1971. Major element variations in ocean-floor basalts: Roy. Soc. London Phil. Trans., A, v. 268, p. 495.
Churkin, M. and Packham, G.H., 1973. Volcanic rocks and volcanic constituents in sediments, Leg 21, Deep Sea Drilling Project. In Burns, R.E., Andrews, J.E., et al., Initial Reports of the Deep Sea Drilling Project, Volume 21: Washington (U.S. Government Printing Office), p. 481.

Coombs, D.S., 1963. Trends and affinities of basaltic magmas and pyroxenes as illustrated on the diopside-olivine-silica diagram: Min. Soc. Am. Spec. Paper, v. 1, p. 227.

Engel, A.E.J., Engel, C.G., and Havens, R.G., 1965. Chemical characteristics of oceanic basalts and the upper mantle: Geol. Soc. Am. Bull., v. 76, p. 719.

Fleischer, M., 1968. Variation of the ratio $\mathrm{Ni} / \mathrm{Co}$ in igneous rock series: J. Wash. Acad. Sci., v. 58, p. 108.

Frey, F.A., Haskin, M.A., Poetz, J.A., and Haskin, L.A., 1968. Rare earth abundances in some basic rocks: J. Geophys. Res., v. 73, p. 6085.

Garrison, R.E., Hein, J.R., and Anderson, T.F., 1973. Lithified carbonate sediment and zeolitic tuff in basalts, Mid-Atlantic Ridge: Sedimentology, v. 20, p. 399.

Gast, P.W., 1968. Trace element fractionation and the origin of tholeiitic and alkaline magma types: Geochim. Cosmochim. Acta, v. 32, p. 1057.

Gottfried, D., Rowe, J.J., and Tilling, R.I., 1972. Distribution of gold in igneous rocks: U.S. Geol. Surv. Prof. Paper 727.

Greenland, L.P. and Fones, R., 1971. Geochemical behavior of silver in a differentiated tholeiitic dolerite sheet: Neues Jahrb. Miner. Mh., h. 9, p. 393.

Hart, S.R., Yeats, R.S., et at., 1974. Oceanic basalt and the Nazca Plate: Geotimes, v. 19, p. 20.

Hart, S.R., Erlauk, A.J., and Kable, E.J.D., 1974. Sea floor basalt alteration: some chemical and $\mathrm{Sr}$ isotopic effects: Contrib. Min. Petrol., v. 44, p. 219.

Irvine, T.N. and Baragar, W.R.A., 1971. A guide to the chemical classification of the common volcanic rocks: Canadian J. Earth Sci., v. 8, p. 523.

Kay, N., Hubbard, N.J., and Gast, P.W., 1970. Chemical characteristics and origin of oceanic ridge volcanic rocks: J. Geophys. Res., v. 75, p. 1585.

Kempe, D.R.C., 1973. Basalts from the southern Indian Ocean: DSDP Leg 26: EOS, Am. Geophys. Union Trans. 54 , p. 1008

Laughton, A.S., Berggren, W.A., et al., 1972. Explanatory notes. In Laughton, A.S., et al., Initial Reports of the Deep Sea Drilling Project, Volume 12: Washington (U.S. Government Printing Office).

Macdonald, G.A., 1968. Composition and origin of Hawaiian lavas: Geol. Soc. Am. Mem., v. 116, p. 477.

Macdougall, D., 1973. Fission track dating of oceanic basalts: EOS, Am. Geophys. Union Trans. 54, p. 987.

Mawson, D., 1950. Basaltic lavas of the Balleny Islands, A.N.A.R.E. report: Roy. Soc. South Australia Trans., v. 73, pt. 2, p. 223.

McKenzie, D. and Sclater, J.G.,.1971. The evolution of the Indian Ocean since the Late Cretaceous: Geophys. J. Roy. Astro. Soc., v. 25, p. 437.

Melson, W.G., 1973. Basaltic glasses from the Deep Sea Drilling Project, chemical characteristics, compositions of alterations products, and fission track "ages": EOS, Am. Geophys. Union Trans. 54, p. 1011.

Miyashiro, A., Shido, F., and Ewing, M., 1969. Diversity and origin of abyssal tholeiite from the Mid-Atlantic Ridge near $24^{\circ}$ and $30^{\circ}$ North latitude: Contrib. Min. Petrol., v. 23 , p. 38.

Moore, J.G., 1970. Water content of basalt erupted on the ocean floor: Contrib. Min. Petrol., v. 28, p. 272.

Moore, J.G. and Fabbi, B.P., 1971. An estimate of the juvenile sulfur content of basalt: Contrib. Min. Petrol., v. 33, p. 118. 
Pearce, J.A. and Cann, J.R., 1973. Tectonic setting of basic volcanic rocks determined using trace element analyses: Earth Planet. Sci. Lett., v. 19, p. 290.

Prinz, M., 1967. Geochemistry of basaltic rocks: trace elements. In Hess, H.H. and Poldervaart, A. (Eds.), Basalts, v. 1,: New York (John Wiley), p. 271.

Salisbury, M.H. and Christensen, N.I., 1973. Progressive weathering of submarine basalt with age: further evidence of sea-floor spreading: Geology, v. 1, p. 63.

Shido, F., Miyashiro, A., and Ewing, M., 1971. Crystallization of abyssal tholeiites: Contrib. Min. Petrol., v. 31, p. 251.

Thompson, G., 1973. A geochemical study of the lowtemperature interaction of seawater and oceanic igneous rocks: EOS, Am. Geophys. Union Trans. 54, p. 1015.

Thompson, G., Bryan, W.B., Frey, F.A., and Sung, C.M. 1974. Petrology and geochemistry of basalts and related rocks from Sites 214, 215, 216, DSDP Leg 22, Indian Ocean. In von der Borch, C.C., Sclater, J.G., et al., Initial
Reports of the Deep Sea Drilling Project, Volume 22: Washington (U.S. Government Printing Office), p. 459.

Thornton, C.P. and Tuttle, O.F., 1960. Chemistry of igneous rocks I. Differentiation index: Am. J. Sci. v. 258, p. 664.

Tobi, A.C., 1963. Plagioclase determination with the aid of the extinction angles in sections normal to (010)-A critical comparison of current albite-Carlsbad charts: Am. J. Sci. v. 261 , p. 157.

Uruno, K., 1963. Optical study on the ordering degree of plagioclases: Sci. Rept. Tohoku Univ., 3rd Ser., v. 8, p. 171.

Weissel, J.K. and Hayes, D.E., 1972. Magnetic anomalies in the southeast Indian Ocean. In Hayes, D.E., (Ed.), Antarctic oceanology II: the Australian-New Zealand sector: Washington, (Am. Geophys. Union, Antarctic Research Series), Vol. 19, p. 165-196.

Yoder, H.S, and Tilley, C.E., 1962. Origin of basalt magmas: an experimental study of natural and synthetic rock systems: J. Petrol. v. 3, p. 342. 